\title{
Clique-circulants and the stable set polytope of fuzzy circular interval graphs
}

\author{
Gianpaolo Oriolo • Gautier Stauffer
}

Received: 12 May 2006 / Accepted: 15 June 2007 / Published online: 5 September 2007

(C) Springer-Verlag 2007

\begin{abstract}
In this paper, we give a complete and explicit description of the rank facets of the stable set polytope for a class of claw-free graphs, recently introduced by Chudnovsky and Seymour (Proceedings of the Bristish Combinatorial Conference, 2005), called fuzzy circular interval graphs. The result builds upon the characterization of minimal rank facets for claw-free graphs by Galluccio and Sassano (J. Combinatorial Theory 69:1-38, 2005) and upon the introduction of a superclass of circulant graphs that are called clique-circulants. The new class of graphs is invetigated in depth. We characterize which clique-circulants $C$ are facet producing, i.e. are such that $\sum_{v \in V(C)} x_{v} \leq \alpha(C)$ is a facet of $S T A B(C)$, thus extending a result of Trotter (Discrete Math. 12:373-388, 1975) for circulants. We show that a simple clique family inequality (Oriolo, Discrete Appl. Math. 132(2):185-201, 2004) may be associated with each clique-circulant $C \subseteq G$, when $G$ is fuzzy circular interval. We show that these inequalities provide all the rank facets of $S T A B(G)$, if $G$ is a fuzzy circular interval graph. Moreover we conjecture that, in this case, they also provide all the non-rank facets of $S T A B(G)$ and offer evidences for this conjecture.
\end{abstract}

Keywords Matching polytope $\cdot$ Stable set polytope $\cdot$ Quasi-line graphs · Circulant graphs

Mathematics Subject Classification (2000) 90C57

The second author was sponsored by the Swiss National Science Foundation.

G. Oriolo

Dipartimento di Ingegneria dell'Impresa, University Tor Vergata, Rome, Italy

e-mail: oriolo@disp.uniroma2.it

G. Stauffer $(\bowtie)$

MIT, room 2-244, Cambridge MA 02139, USA

e-mail: gautier.stauffer@gmail.com 


\section{Preliminary}

In this paper, we deal with simple and undirected graphs $G$ with vertex set $V(G)$ and edge set $E(G)$. If $H$ is a subgraph of $G$, then we write $H \subseteq G$. We denote by $\alpha(G)$ the stability number of $G$. If $v \in V(G)$, we denote by $N(v)$ the neighborhood of $v$, the set of vertices that are adjacent to $v$.

Let $W$ be a subset of $V(G)$. We denote by $G[W]$ the subgraph of $G$ induced by $W$. $G[W]$ is $\alpha$-maximal if $\alpha(G[W \cup\{v\}])>\alpha(G[W])$ for every $v \notin W$. We denote by $N(W)$ the neighborhood of $W$, that is, the set of vertices not in $W$ that are adjacent to at least one vertex of $W$, that is, $N(W)=\{u \in V(G) \backslash W: \exists v \in W, u v \in E\}$; we denote by $\Gamma(W)$ the set of vertices in $N(W)$ that are adjacent to all vertices of $W$, that is, $\Gamma(W)=\{u \in V(G) \backslash W: u v \in E, \forall v \in W\}$. Sometimes, if $H$ is a subgraph of $G$, we simply write $N(H)$ for $N(V(H))$ and $\Gamma(H)$ for $\Gamma(V(H))$. If $X \subseteq \Gamma(W)$ we say that $W$ and $X$ are complete.

\section{Introduction}

The matching polytope $M A T C H(G)$ is the convex hull of the characteristic vectors of matchings of the graph $G$. The matching problem is a well known example of a combinatorial optimization problem in which the optimization problem $[5,6]$ on the one hand and the polyhedral structure, i.e. the facets of $\operatorname{MATCH}(G)$, on the other hand are well understood [5].

A graph $G$ is claw-free if no vertex has three pairwise nonadjacent neighbors. Line graphs are claw free and thus the weighted stable set problem for a claw-free graph is a generalization of the weighted matching problem of a graph. While the general stable set problem is NP-complete, it can be solved in polynomial time on a claw-free graph $[12,20]$ even in the weighted case $[14,15]$ see also [21]. These algorithms are extensions of Edmonds' matching algorithms.

The stable set polytope $S T A B(G)$ is the convex hull of the characteristic vectors of stable sets of the graph $G$. We say that a graph $G$ is facet-defining if the inequality $\sum_{v \in V(G)} x_{v} \leq \alpha(G)$ is a facet of $S T A B(G)$. In spite of the knowledge of polynomial time algorithms for finding maximum weighted stable sets, and results characterizing the rank-facets [8] (facets with 0/1 normal vectors) of claw-free graphs, or giving a compact lifted formulation for the subclass of distance claw-free graphs [18], the problem of finding a “...decent linear description of the stable set polytope" [10] of a claw-free graph is a long-time open question.

Such a result exists for the class of quasi-line graphs. A graph is quasi-line if the neighborhood of any vertex partitions into two cliques. Trivially, we have the following:

Fact 1 Let $G$ be a quasi-line graph and $Q$ a subset of $V(G) . \Gamma(Q)$ partitions into two cliques.

Quasi-line graphs are a superclass of line graphs and a subclass of claw-free graphs. The complement of quasi-line graphs are called near-bipartite and their stable set polytope has been studied in $[13,22]$. The problem of giving a linear description of 
$S T A B(G)$ when $G$ is quasi-line was first considered by Ben Rebea [19]. Oriolo [16] formulated a conjecture, inspired by the work of Ben Rebea, that is based on the so-called clique family inequalities.

Clique family inequalities have been introduced in [16], where the following theorem is proved. Let $\mathcal{F}$ be a set of $n$ cliques of $G, 1 \leq p \leq n$ be integral and $r=n$ $\bmod p$. Let $V_{p-1}(\mathcal{F})$ the set of vertices of $G$ covered by $(p-1)$ cliques of $\mathcal{F}$ and $V_{\geq p}(\mathcal{F})$ the set of vertices of $G$ covered by $p$ or more cliques of $\mathcal{F}$.

Theorem 1 [16] The clique family inequality associated with $\mathcal{F}$ and $p$

$$
(p-r) \cdot \sum_{v \in V_{\geq p}(\mathcal{F})} x_{v}+(p-r-1) \cdot \sum_{v \in V_{p-1}(\mathcal{F})} x_{v} \leq(p-r) \cdot\left\lfloor\frac{n}{p}\right\rfloor
$$

is valid for $S T A B(G)$.

Conjecture 1 (Ben Rebea conjecture [16]) The stable set polytope of a quasi-line graph $G=(V, E)$ may be described by the following inequalities:

(i) $x(v) \geq 0$ for each $v \in V$

(ii) $\sum_{v \in K} x(v) \leq 1$ for each maximal clique $K$

(iii) inequalities (1) for each family $\mathcal{F}$ of maximal cliques and each integer $p$ with $|\mathcal{F}|>2 p \geq 4$ and $|\mathcal{F}| \bmod p \neq 0$.

Ben Rebea conjecture has been proved in two steps. First, Chudnovsky and Seymour provided a decomposition result for quasi-line graphs and proved that the Ben Rebea conjecture holds, if the quasi-line graph is not a fuzzy circular interval graph [3]. Then, Eisenbrand, Oriolo, Stauffer and Ventura [7] proved that the Ben Rebea conjecture holds for fuzzy circular interval graphs too. Fuzzy circular interval graphs are a subclass of quasi-line graphs and they will be defined in Sect. 2. It is very interesting to point out that that quasi-line graphs that are not fuzzy circular interval graph have only rank facets and these facets have a matching-like structure (in fact, they are called Edmonds inequalities [3]).

While Ben Rebea Theorem gives an algebraic characterization of the facets of quasiline graphs, we would like to understand their combinatorial structure. Understanding the combinatorial structure could lead, for instance, to devise efficient algorithms for the separation of the corresponding inequalities.

In this paper, we study the stable set polytope of fuzzy circular interval graphs and focus in particular on the rank facets. We first define this class of graphs and some of their properties in Sect. 2. We then introduce the class of clique-circulants which defines the core of rank facets for fuzzy circular interval graphs. They are defined as follows:

Definition 1 A quasi-line graph $G(V, E)$ is an $(n, p)$-clique-circulant if the following statements hold:

(i) There exists a partition of $V(G)$ into $n$ non-empty cliques $Q_{1}, \ldots, Q_{n}$ and an integer $p$, with $n \geq 2 p \geq 4$, such that $\Gamma\left(Q_{i}\right) \supseteq Q_{i-p+1} \cup \cdots \cup Q_{i-1} \cup Q_{i+1} \cup$ $\cdots \cup Q_{i+p-1}$, for $i=1, \ldots, n$. 
Fig. 1 A $(5,2)$-clique-circulant

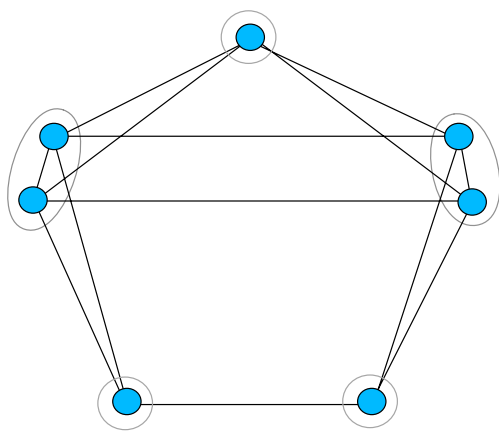

(ii) There exists an $(n, p)$-circulant $W=\left\{v_{1}, \ldots, v_{n}\right\}$ with $v_{i} \in Q_{i}$ for $i=1, \ldots, n$.

A $(5,2)$-clique-circulant is depicted in Fig. 1.

Clique circulants are invetigated in depth. They are a superclass of circulants, also called antiwebs, a well known class of graphs with interesting polyhedral properties $[1,2,23]$. We show that an $(n, p)$ clique-circulant $C$ is facet producing, i.e. such that $\sum_{v \in V(C)} x_{v} \leq \alpha(C)$ is a facet of $S T A B(C)$, if and only if $p$ is not a divisor of $n$ (cf. Theorem 4), thus extending a result of Trotter for circulants [23].

Our main result, an explicit description of all the rank facets of the stable set polytope of fuzzy circular interval graphs, builds upon the characterization of minimal rank facets for claw-free graphs by Galluccio and Sassano [8] and upon the introduction of a a subclass of clique-circulants, tight clique-circulants, that are defined and investigated in Sect. 4. Those graphs are closely related to clique-circulants, see Sect. 4.3, but have many additional properties that we will exploit in the constructive proof of our result.

The result is given in Sect. 5: if $\sum_{j \in V\left(G^{\prime}\right)} x_{j} \leq \alpha\left(G^{\prime}\right)$ is a facet of $S T A B(G)$, with $G^{\prime} \subseteq G$ and $G$ fuzzy circular interval, then $G^{\prime}$ is either a maximal clique or an $\alpha$-maximal $(n, p)$ clique-circulant with $n \bmod p \neq 0$.

We also show that, when $G$ is fuzzy circular interval, a simple clique family inequality may be associated with each clique-circulant $C \subseteq G$ (cf. Corollary 4). These inequalities are in general non-rank, but we show that they do produce all the the rank facets of $S T A B(G)$. In fact, these inequalities also produce all the non-rank facets of $S T A B(G)$ that are known so far $[9,11,16]$, we therefore close with the conjecture (cf. Conjecture 2) that they provide all the facets, i.e. rank and non-rank, of $S T A B(G)$, when $G$ is fuzzy circular interval.

$N B$ : The reader interested in the main flavour of this paper does not need to go into the details of Sect. 4, where tight clique-circulants are defined and investigated.

\section{Fuzzy circular interval graphs}

Chudnovsky and Seymour [3] proved the following structural result about quasi-line graphs. 
Theorem 2 [3] A connected quasi-line graph $G$ is either a fuzzy circular interval graph, or it is the composition of fuzzy linear interval strips with a collection of disjoint cliques.

The definition of fuzzy linear interval strips can be found in [3], while the definition of fuzzy circular interval graphs is given later in the following. First, we need the definition of circular interval graph

Definition 2 A circular interval graph [3] $G=(V, E)$ is defined by the following construction: Take a circle $\mathcal{C}$ and a set of vertices $V$ on the circle. Take a subset of intervals $\mathcal{I}$ of $\mathcal{C}$ and say that $u, v \in V$ are adjacent if $\{u, v\}$ is a subset of one of the intervals.

From the definition, it is quite easy to observe that circular interval graphs are quasi-line. Fuzzy circular interval graphs are a superclass of circular interval graphs.

Definition 3 A graph $G=(V, E)$ is fuzzy circular interval if the following conditions hold.

(i) There is a map $\Phi$ from $V$ to a circle $\mathcal{C}$.

(ii) There is a set of intervals $\mathcal{I}$ of $\mathcal{C}$, none including another, such that no point of $C$ is the end of more than one interval so that:

(a) If two vertices $u$ and $v$ are adjacent, then $\Phi(u)$ and $\Phi(v)$ belong to a common interval.

(b) If two vertices $u$ and $v$ belong to the same interval, which is not an interval with endpoints $\Phi(u)$ and $\Phi(v)$, then they are adjacent.

In other words, in a fuzzy circular interval graph, adjacencies are completely described by the pair $(\Phi, \mathcal{I})$, except for vertices $u$ and $v$ such that one of the intervals with endpoints $\Phi(u)$ and $\Phi(v)$ belongs to $\mathcal{I}$. For these vertices adjacency is fuzzy. When considering a fuzzy circular interval graph, we often consider a representation $(\Phi, \mathcal{I})$ and we detail the fuzzy adjacencies only when needed. Sometimes, we abuse notation and denote by $G=(V, \Phi, \mathcal{I})$ (or even $G=(\Phi, \mathcal{I})$ ) a fuzzy circular interval graph with vertex set $V$ and representation $(\Phi, \mathcal{I})$.

It is easy to see that fuzzy circular interval graphs are a superclass of circular interval graphs, but they are a subclass of quasi-line graphs. The following lemma is from [7]. A graph is $C_{4}$-free if it does not have an induced subgraph isomorphic to a cordless cycle of length 4 .

Lemma 1 [7] If $G$ is $C_{4}$-free, then it is fuzzy circular interval if and only if it is circular interval.

A $n e t$ is a graph isomorphic to the graph with vertex set $\left\{u_{1}, u_{2}, u_{3}, v_{1}, v_{2}, v_{3}\right\}$ and edge set $\left\{\left(u_{1}, u_{2}\right),\left(u_{2}, u_{3}\right),\left(u_{3}, u_{1}\right),\left(u_{1}, v_{1}\right),\left(u_{2}, v_{2}\right),\left(u_{3}, v_{3}\right)\right\}$. A graph is net-free if it does not have an induced subgraph isomorphic to a net. We omit the proof of the next simple lemma.

Lemma 2 A net is not a circular interval graph. 
Corollary 1 Fuzzy circular interval graphs are net-free.

Proof By definition, if $G$ is a fuzzy circular interval graph and $H$ is an induced subgraph, then $H$ is a fuzzy circular interval graph too. On the other hand, a net $H$ is not a fuzzy circular interval graph: this follows from Lemma 1 and 2.

A Hajos graph is a graph isomorphic to the graph with vertex set $\left\{u_{1}, u_{2}, u_{3}\right.$, $\left.v_{1}, v_{2}, v_{3}\right\}$ and edge set $\left\{\left(u_{1}, u_{2}\right),\left(u_{2}, u_{3}\right),\left(u_{3}, u_{1}\right),\left(u_{1}, v_{2}\right),\left(u_{1}, v_{3}\right),\left(u_{2}, v_{1}\right)\right.$, $\left.\left(u_{2}, v_{3}\right),\left(u_{3}, v_{1}\right),\left(u_{3}, v_{2}\right)\right\}$. The set of vertices $\left\{u_{1}, u_{2}, u_{3}\right\}$ is the core of the Hajos graph. Given a graph $G=(V, E)$, a set of vertices $S \subseteq V$ is dominating if every vertex of $V \backslash S$ is adjacent to a vertex of $S$.

Lemma 3 Let $G$ be a fuzzy circular interval graph and let $H \subseteq G$ be an Hajos subgraph of $G$. The core of $H$ is a dominating set for $G$.

Proof Let $V(H)=\left\{u_{1}, u_{2}, u_{3}, v_{1}, v_{2}, v_{3}\right\}$ with adjacencies as above. Suppose that $\left\{u_{1}, u_{2}, u_{3}\right\}$ is not a dominating set. Hence there exists $z \notin V(H)$ such $z u_{i} \notin E$, $i=1 . .3$.

If $z$ is not adjacent to any vertex of $V(H)$, then $G[V(H) \cup z]$ is $C_{4}$-free. It is easy to check that $G[V(H) \cup z]$ is not a circular interval graph and then we are done by Lemma 1. If $z$ is adjacent to a single vertex in $V(H)$, that is, in $\left\{v_{1}, v_{2}, v_{3}\right\}$, then $G[V(H) \cup z]$ has a net and we are done by Corollary 1 . If $z$ is adjacent to $v_{1}, v_{2}$ and $v_{3}$ then there is a claw. The last case is when $z$ is adjacent to two vertices in $\left\{v_{1}, v_{2}, v_{3}\right\}$ : a careful analysis of the possible cases rules out this case as well.

\section{A good candidate for the rank facets : clique-circulants}

Circulant graphs (or antiweb) were introduced by Trotter [23].

Definition 4 [23] Let $n, p \in \mathbb{N}$ with $n \geq 2 p$. A ( $n, p)$-circulant $W$ is the graph with vertices $V(W)=\{1,2, \ldots, n\}$ and edges $E(W)=\{(i, j): 1 \leq i, j \leq n$ and $\mid i-$ $j \mid \leq p-1\}$.

Given a $(n, p)$-circulant $W$, we can associated a valid inequality for $S T A B(W)$ : $\sum_{j \in V(W)} x_{j} \leq \alpha(W)$. Trotter studied when this inequality is facet-defining for $S T A B(W)$.

Theorem 3 [23] If $W$ is an $(n, p)$-circulant, then $\alpha(W)=\left\lfloor\frac{n}{p}\right\rfloor$ and $\sum_{j \in W} x_{j} \leq$ $\alpha(W)$ is a facet of $S T A B(W)$ if and only if $p$ is not a divisor of $n$.

In [17] Padberg proved the following. Let $G=(V, E)$ be a graph and $\sum_{j \in T} a_{j} x_{j} \leq b$ be a facet of $S T A B(G[T])$, for some $T \subset V$. If $u \in V \backslash T$ and

$$
a_{u}=b-\max _{S \text { stable set, } S \subseteq T \backslash N(u)} \sum_{j \in S} a_{j},
$$

then the inequality $\sum_{j \in T \cup u} a_{j} x_{j} \leq b$ is a facet of $S T A B(G[T \cup u])$ and $a_{u}$ is the lifting coefficient of variable $x_{u}$. Of course, we can iterate this procedure and, after 
$|V \backslash T|$ steps, produce an inequality $\sum_{j \in V} a_{j} x_{j} \leq b$ which is a facet of $S T A B(G)$ and is a sequential lifting of $\sum_{j \in T} a_{j} x_{j} \leq b$.

By instantiating a result by Galluccio and Sassano [8] for claw-free graphs to fuzzy circular interval graphs, it is possible to prove the following (see Appendix A).

Lemma 4 Let $G=(V, E)$ be a fuzzy circular interval graph. Every rank facet can be obtained by sequential lifting of an inequality $\sum_{v \in V\left(G^{\prime}\right)} x_{v} \leq \alpha\left(G^{\prime}\right)$ where

(i) $G^{\prime}$ is a singleton;

(ii) $G^{\prime}$ is $a(\alpha \omega+1, \omega)$-circulant, $\omega \geq 2$.

This proves that, beside cliques, circulant graphs form the core of the rank facets for the SSP of fuzzy circular interval graphs. Oriolo [16] showed that the rank facets of the stable set polytope of quasi-line graphs have the following property.

Lemma 5 [16] Let $G=(V, E)$ be a quasi-line graph and $Q$ a subset of vertices. The inequality $\sum_{j \in Q} x_{j} \leq \alpha(Q)$ is a facet of $S T A B(G)$ if and only if the following statements hold:

(i) $\sum_{j \in Q} x_{j} \leq \alpha(Q)$ defines a facet of $S T A B(G[Q])$;

(ii) $G[Q]$ is $\alpha$-maximal.

Given a fuzzy circular interval graph $G$, we are interested in describing the induced subgraph of $G$ which are facet-defining (like cliques for instance). The previous Lemma tells us that those structures must be invariant under adding vertices that do not increase the stability number (e.g duplication of a vertex). Circulant graphs do not have this property but since they form the core of the rank facets, it is natural to try to find a super-class of them with the same facet-defining properties but with the additional invariant characteristic. This is the case of clique-circulant graphs.

Definition 5 Let $G$ be a graph, $Q_{1}, \ldots, Q_{n}$ a collection of non-empty cliques that partition $V(G)$ and $p>1$ an integer such that $n \geq 2 p$. The cliques $Q_{1}, \ldots, Q_{n}$ define an $(n, p)$-clique-partition of $V(G)$ if $\Gamma\left(Q_{i}\right) \supseteq Q_{i-p+1} \cup \cdots \cup Q_{i-1} \cup Q_{i+1} \cup \cdots \cup$ $Q_{i+p-1}$ for $i=1, \ldots, n$.

Definition 6 A quasi-line graph $G$ is an $(n, p)$-clique-circulant if the following statements hold:

(i) There exists an $(n, p)$-clique-partition $Q=\left\{Q_{i}, i=1, \ldots, n\right\}$ of $V(G)$.

(ii) There exists an $(n, p)$-circulant $W=\left\{v_{1}, \ldots, v_{n}\right\}$, with $v_{i} \in Q_{i}$ for $i=1, \ldots, n$.

A $(5,2)$-clique-circulant is depicted in Fig. 1. Observe that it follows from the definition that, for some $i$ and $j$ with $|i-j| \geq p$, there might be edges between $Q_{i}$ and $Q_{j}$, but it is not possible that a vertex in $Q_{i}\left(Q_{j}\right)$ is totally joined to $Q_{j}\left(Q_{i}\right)$.

The following results show that clique-circulants generalize circulant graphs from both graph theoretical and polyhedral point of views.

Lemma 6 An $(n, p)$-circulant $C=\{1, \ldots, n\}$ with $n \geq 2 p$ is an $(n, p)$-cliquecirculant. 
Proof Just observe that $C=\{\{i\}, i=1, \ldots, n\}$ is an $(n, p)$-clique-partition of $V(C)$.

Corollary 2 If $G$ is an $(n, p)$-clique-circulant, then $\alpha(G)=\left\lfloor\frac{n}{p}\right\rfloor$.

Proof If $Q=\left\{Q_{i}, i=1, \ldots, n\right\}$ defines an $(n, p)$-clique-partition of $V(G)$, then $K_{i}:=Q_{i} \cup \cdots \cup Q_{i+p-1}$ is a clique, for $i=1 . . n$. Every vertex of $G$ is then covered by at least $p$ cliques of the family $\left\{K_{i}, i=1, \ldots, n\right\}$ and therefore, $\alpha(G) \leq\left\lfloor\frac{n}{p}\right\rfloor$ holds. On the other hand, since there exists an $(n, p)$-circulant $W=\left\{v_{1}, \ldots, v_{n}\right\}$, with $v_{i} \in Q_{i}$ for $i=1, \ldots, n$, we have that $\alpha(G) \geq\left\lfloor\frac{n}{p}\right\rfloor$.

We can extend Theorem 3 to clique-circulants.

Theorem 4 Let $G$ be an (n,p)-clique-circulant. Then $\alpha(G)=\left\lfloor\frac{n}{p}\right\rfloor$ and $\sum_{j \in V(G)} x_{j} \leq\left\lfloor\frac{n}{p}\right\rfloor$ is a facet of $S T A B(G)$ if and only if $p$ is not a divisor of $n$.

Proof The inequality is valid from Corollary 2. Let $Q=\left\{Q_{i}, i=1, \ldots, n\right\}$ be an $(n, p)$-clique-partition of $V(G)$ and $W=\left\{v_{1}, \ldots, v_{n}\right\}$ an $(n, p)$-circulant, with $v_{i} \in Q_{i}$ for $i=1, \ldots, n$.

Necessity. Suppose that $n=q p$ for some integer $q$; it follows that $\left\lfloor\frac{n}{p}\right\rfloor=q$. Since $Q_{1}, \ldots, Q_{n}$ define an $(n, p)$-clique-partition of $V(G)$, then, for $i=1, \ldots, q$, $K_{i}:=\bigcup_{j=1, \ldots, p} Q_{(i-1) p+j}$ is a clique. Then the inequality $\sum_{j \in V(Q)} x_{j} \leq q$ is not a facet of $S T A B(G)$, since it can be obtained as the sum of the inequalities $\sum_{j \in K_{i}} x_{j} \leq 1$.

Sufficiency. Since $p$ is not a divisor of $n$, the inequality $\sum_{j \in V(W)} x_{j} \leq \alpha(W)$ is a facet of $S T A B(G[W])$ by Theorem 3. Finally, since $\alpha(G)=\alpha(W), \sum_{j \in V(G)} x_{j} \leq$ $\alpha(G)$ is a facet of $S T A B(G)$ by Lemma 5 .

Again, a crucial fact for our analysis is that, when dealing with fuzzy circular interval graphs, clique-circulants are invariant with respect to the addition of vertices that do not increase the stability number. In fact, we have the following theorem:

Theorem 5 Let $G(V, E)$ be a fuzzy circular interval graph, $Q \subset V$ such that $G[Q]$ is an $(n, p)$-clique-circulant and $v \notin Q$ such that $\alpha(Q \cup\{v\})=\alpha(Q)$. Then $G[Q \cup\{v\}]$ is an $\left(n^{\prime}, p^{\prime}\right)$-clique-circulant, with $\left\lfloor\frac{n^{\prime}}{p^{\prime}}\right\rfloor=\left\lfloor\frac{n}{p}\right\rfloor$.

The theorem is illustrated in Fig. 2. A $(5,2)$-clique-circulant $C$ and a vertex $v$, that does not increase the stability number, are drawn in the left picture. The right picture shows that $G[V(C) \cup\{v\}]$ is indeed a $(8,3)$-clique-circulant.

The proof of Theorem 5 is constructive and will be given later since it is based on the properties of tight clique-circulants, a class that will be defined in the next section.

\section{On a constructive proof, a useful class: tight Clique-Circulants}

Clique-circulant have a couple of nice properties as we have observed in the previous Section. Nevertheless in our constructive proof, we needed some additional properties of circulants that clique-circulants do not share. We therefore needed to define a closely related subclass: tight clique-circulants. 

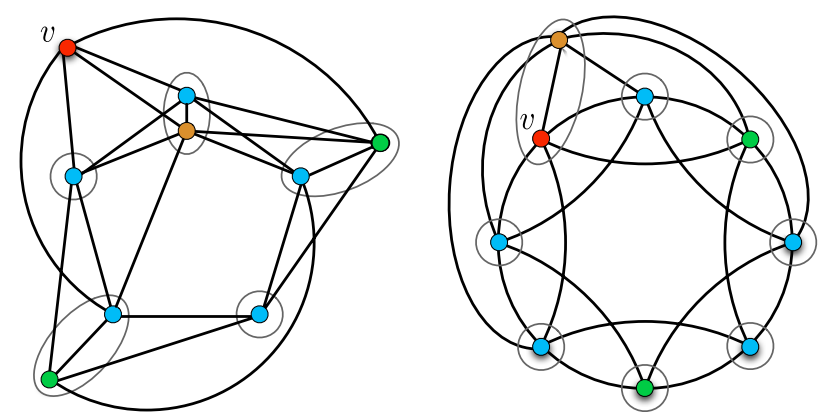

Fig. 2 Clique-circulants are invariant with respect to the addition of vertices that do not increase the stability number

Definition 7 Let $G$ be a graph, and $C_{1}, \ldots, C_{n}$ an $(n, p)$-clique-partition of $V(G)$. $C_{1}, \ldots, C_{n}$ define a tight $(n, p)$-clique-partition if the following statements hold:

- $\Gamma\left(C_{i}\right)=C_{i-p+1} \cup \cdots \cup C_{i-1} \cup C_{i+1} \cup \cdots \cup C_{i+p-1}$ for $i=1, \ldots, n$.

- For $1 \leq i \leq n$, there is a unique partition of $\Gamma\left(C_{i}\right)$ into two cliques. Namely, they are: $C_{i-p+1} \cup \cdots \cup C_{i-1}$ and $C_{i+1} \cup \cdots \cup C_{i+p-1}$.

Recognizing whether a clique-partition $C_{1}, \ldots, C_{n}$ of $V(G)$ is tight is easy. In particular, the partition of $\Gamma\left(C_{i}\right)$ into two cliques is unique if and only if the complement of $G\left[\Gamma\left(C_{i}\right)\right]$ is bipartite and connected. For instance: the $(5,2)$-clique-partition in Fig. 1 is not tight; the $(5,2)$-clique-partition in Fig. 2 is tight; the $(8,3)$-clique-partition in Fig. 2 is not.

We will later show the following fact:

Lemma 7 Let $G$ be a quasi-line graph and $C=\left\{C_{i}, i=1, \ldots, n\right\}$ a tight $(n, p)$ clique-partition of $V(G)$. There exists $W=\left\{v_{i}, i=1, \ldots, n\right\}$, with $v_{i} \in C_{i}$ for $i=1, \ldots, n$, such that $G[W]$ is an $(n, p)$-circulant.

The previous lemma motivates the following extension of Definition 6:

Definition 8 A quasi-line graph $G=(V, E)$ is a tight $(n, p)$-clique-circulant if there exists a tight $(n, p)$-clique-partition $\left\{C_{i}, i=1, \ldots, n\right\}$ of $V$.

Throughout the paper, when we refer to a tight $(n, p)$-clique-circulant $C=\left\{C_{i}, i=\right.$ $1, \ldots, n\}$, we mean that $C_{1}, \ldots, C_{n}$ define a tight $(n, p)$-clique-partition of the vertices of a quasi-line graph $C$.

Trivially, the $(5,2)$-clique-circulant in Fig. 1 is not tight. Vice versa, each $(n, p)$ circulant with $n>2 p$ is a tight $(n, p)$-clique-circulant.

Lemma 8 An $(n, p)$-circulant $C=\{1, \ldots, n\}$ with $n>2 p$ is a tight $(n, p)$-cliquecirculant.

Proof Just observe that $C=\{\{i\}, i=1, \ldots, n\}$ is a tight $(n, p)$-clique-partition of $V(C)$.

The proof of Lemma 7 requires the following: 
Lemma 9 Let $G$ be a quasi-line graph, $C=\left\{C_{i}, i=1, \ldots, n\right\}$ a tight $(n, p)$-cliquepartition of $V(G)$ and $v \in C_{i}$ for some $i \in\{1, \ldots, n\}$. The following statements hold:

(i) $N(v) \subset C_{i-p} \cup \cdots \cup C_{i-1} \cup\left(C_{i} \backslash v\right) \cup C_{i+1} \cup \cdots \cup C_{i+p}$

(ii) For any partition of $N(v)$ into two cliques $K_{l}, K_{r}$, without loss of generality:

$$
\begin{gathered}
\left(N(v) \cap C_{i-p}\right) \cup C_{i-p+1} \cup \cdots \cup C_{i-1} \subseteq K_{l} \text { and } \\
C_{i+1} \cup \cdots \cup C_{i+p-1} \cup\left(N(v) \cap C_{i+p}\right) \subseteq K_{r} .
\end{gathered}
$$

Proof Since $C$ is quasi-line, $N(v)$ can be partitioned into two cliques $K_{l}$ and $K_{r}$ (see Fact 1). Since $C_{1}, \ldots, C_{n}$ define a tight $(n, p)$-clique-partition, there exists a unique partition of $\Gamma\left(C_{i}\right)$ into two cliques $\Gamma^{-}\left(C_{i}\right)=C_{i-p+1} \cup \cdots \cup C_{i-1}$ and $\Gamma^{+}\left(C_{i}\right)=C_{i+1} \cup \cdots \cup C_{i+p-1}$. Thus, without loss of generality, $\Gamma^{-}\left(C_{i}\right) \subseteq K_{l}$ and $\Gamma^{+}\left(C_{i}\right) \subseteq K_{r}$.

(i) Suppose to the contrary that there exist $u \in C_{j}$ such that $u v \in E$ with $|i-j|>$ $p$. It follows that either $\Gamma^{-}\left(C_{i}\right) \subseteq N(u)$ or $\Gamma^{+}\left(C_{i}\right) \subseteq N(u)$; without loss of generality we assume $\Gamma^{+}\left(C_{i}\right)=\left\{C_{i+1} \cup \cdots \cup C_{i+p-1}\right\} \subseteq N(u)$. Then $u \in \Gamma\left(C_{i+1}\right)$ and, since $u \notin C_{i-p} \cup \cdots \cup C_{i+p}$, we are in contradiction since $\Gamma\left(C_{i+1}\right)=C_{i-p+2} \cup \ldots \cup C_{i} \cup C_{i+2} \cup \cdots \cup C_{i+p}$.

(ii) We know from (i) that $N(v) \subset C_{i-p} \cup \cdots \cup C_{i-1} \cup\left(C_{i} \backslash v\right) \cup C_{i+1} \cup \cdots \cup C_{i+p}$. Since $n>2 p,|(i+1)-(i-p)|>p-1$. Thus, by definition, $C_{i-p} \cap \Gamma\left(C_{i+1}\right)=$ $\emptyset$. Since $C_{i+1} \subseteq K_{r}$, it follows that $\left(N(v) \cap C_{i-p}\right) \subseteq K_{l}$. By a similar argument $\left(N(v) \cap C_{i+p}\right) \subseteq K_{r}$.

Proof of Lemma 7 In the following, for convenience, we extend the definition of an $(n, p)$-circulant to $p=1$. In that case it is just a stable set of $n$ vertices. Let $d=$ $\operatorname{gcd}(n, p), n^{\prime}=n / d$ and $p^{\prime}=p / d$.

For any $v \in C_{n}$, define the following sets:

$$
\begin{aligned}
& L_{0}(v)=\{v\} ; \\
& L_{q p}(v)=C_{q p} \backslash \Gamma\left(L_{(q-1) p}(v)\right) \text { for } q=1, \ldots, n^{\prime} ;
\end{aligned}
$$

Observe that the sets $L_{q p}(v), q=1, \ldots, n^{\prime}$ are non-empty, else $L_{(q-1) p}(v) \subset$ $\Gamma\left(C_{q p}\right)$ and this would contradict the fact that the set $\left\{C_{i}, i=1, \ldots, n\right\}$ defines a tight $(n, p)$-clique-partition.

Claim 1 There exists $v \in C_{n}$ such that $v \in L_{n^{\prime} p}(v)$.

Proof Suppose to the contrary that for all $v \in C_{n}, v \notin L_{n^{\prime} p}(v)$. We choose $v_{1} \in C_{n}$ such that $\left|L_{n^{\prime} p}\left(v_{1}\right)\right|=\min _{v \in C_{n}}\left|L_{n^{\prime} p}(v)\right|$. We have already observed that $\left|L_{n^{\prime} p}\left(v_{1}\right)\right|>0$. Hence we choose $v_{2} \in L_{n^{\prime} p}\left(v_{1}\right) . v_{2}, L_{\left(n^{\prime}-1\right) p}\left(v_{1}\right)$ and $C_{p} \backslash L_{p}\left(v_{1}\right)$ are in the neighborhood of $v_{1}$. Let $K_{l}\left(v_{1}\right), K_{r}\left(v_{1}\right)$ be the classes of a partition of $N\left(v_{1}\right)$ into two cliques. By statement (ii) of Lemma 9, $C_{p} \backslash L_{p}\left(v_{1}\right) \subseteq K_{r}\left(v_{1}\right)$ and $L_{\left(n^{\prime}-1\right) p} \subseteq K_{l}\left(v_{1}\right)$. Hence $v_{2} \in K_{r}\left(v_{1}\right)$ and thus $L_{p}\left(v_{2}\right) \subseteq L_{p}\left(v_{1}\right)$. As a consequence, $L_{n^{\prime} p}\left(v_{2}\right) \subseteq L_{n^{\prime} p}\left(v_{1}\right)$. But, since $v_{2} \in L_{n^{\prime} p}\left(v_{1}\right)$ and $v_{2} \notin L_{n^{\prime} p}\left(v_{2}\right)$, it follows that $\left|L_{n^{\prime} p}\left(v_{1}\right)\right|>\left|L_{n^{\prime} p}\left(v_{2}\right)\right|$, which is a contradiction. (end of the claim) 
Let $v \in C_{n}$ satisfy the claim. We choose $n^{\prime}$ vertices $\left\{z_{n^{\prime}}, z_{\left(n^{\prime}-1\right) p}, \ldots, z_{p}\right\}$ from $V$ as follows. First, we set $z_{n^{\prime} p}=v$; then for $q=\left(n^{\prime}-1\right)$ down to 1 we choose a vertex $z_{q p} \in L_{q p}(v) \backslash N\left(z_{(q+1) p}\right)$ (this set is non-empty either by definition or because of the claim). It is straightforward to check that $W=C\left[\left\{z_{n^{\prime} p}, z_{\left(n^{\prime}-1\right) p}, \ldots, z_{p}\right\}\right]$ is an $\left(n^{\prime}, p^{\prime}\right)$-circulant.

By a simple rotation, we can repeat the argument above as to conclude that, for any $h \in\{0, \ldots, d-1\}$, there exists an $\left(n^{\prime}, p^{\prime}\right)$-circulant $W_{h}=\left\{z_{q p-h}, q=1, \ldots, n^{\prime}\right\}$, with $z_{q p-h} \in C_{q p-h}$ for each $q$.

Finally, observe that the vertices $z_{i}, i=1, \ldots, n$ are such that $z_{i} \notin N\left(z_{i-p}\right) \cup$ $N\left(z_{i+p}\right)$ for any $i$; moreover $z_{i} z_{j} \notin E$ if $|i-j|>p$, by Lemma 9. Therefore they induce an $(n, p)$-circulant.

\subsection{The neighborhood of tight clique-circulants in fuzzy circular interval graphs}

Several authors [3,8] were interested in the neighborhood of a hole $H \subseteq G$, when $G$ is a quasi-line graph. They observed that, in this case, $N(H)$ partitions into 4 classes, since each vertex $v \in N(H)$ is adjacent to either 2 , or 3 , or 4 vertices of $H$ and, in particular, the following statements hold (see Fig. 3):

- if $v$ is adjacent to 2 vertices of $H$, then these vertices are contiguous on $H$ (vertex $A$ in figure);

- if $v$ is adjacent to 3 vertices of $H$, then these vertices are contiguous on $H$ (vertex $B$ in figure);

- if $v$ is adjacent to 4 vertices of $H$, then either these vertices are contiguous on $H$ (vertex $D$ in figure), or they split into two pairs of contiguous vertices (vertex $E$ in figure).

We point out that, when $G$ is a connected quasi-line graph and $H \subseteq G$ is an hole, in general, there are vertices of $G$ that are not in the neighborhood of $H$ (vertex $F$ in figure). In the following, we show (Theorem 6) that the neighborhood of a tight

Fig. 3 The different types of vertices

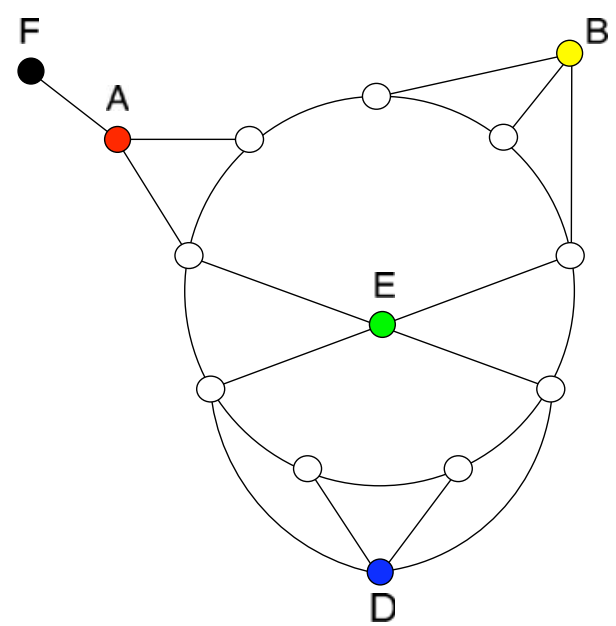


clique-circulant $C \subseteq G$, with $G$ a fuzzy circular interval graph, can be partitioned into 3 classes $A(C), B(C), D(C)$ and that, if $G$ is connected ${ }^{1}$, each vertex of $G$ either belongs to $C$ or to $N(C)$.

Definition 9 Let $G$ be a graph and $C=\left\{C_{i}, i=1, \ldots, n\right\}$ a subgraph of $G$ that is a tight $(n, p)$-clique-circulant. For each $v \notin V(C)$, let $\Gamma(v, C)=\left\{C_{i}, i=1 \ldots n\right.$ : $\left.C_{i} \subseteq N(v)\right\}$ and:

- $A(C)=\left\{v \in N(C): \Gamma(v, C)=\left\{C_{i+1}, \ldots, C_{i+2 p-2}\right\}\right.$ for some $\left.i\right\}$.

- $B(C)=\left\{v \in N(C): \Gamma(v, C)=\left\{C_{i+1}, \ldots, C_{i+2 p-1}\right\}\right.$ for some $\left.i\right\}$.

- $D(C)=\left\{v \in N(C): \Gamma(v, C)=\left\{C_{i+1}, \ldots, C_{i+2 p}\right\}\right.$ for some $\left.i\right\}$.

Before going into the proof of Theorem 6, we state a couple of definitions that we extensively use.

Definition 10 We denote by $\mathcal{C}(n, p)$ the set of graphs that are tight $(n, p)$-cliquecirculants, for given $n$ and $p$. Analogously, for a graph $G$, we denote by $\mathcal{C}_{G}(n, p)$ the set of subgraphs of $G$ that are tight $(n, p)$-clique-circulants.

Let $G$ be a quasi-line graph and let $C=\left\{C_{i}, i=1, \ldots, n\right\} \in \mathcal{C}_{G}(n, p)$. Since $G$ is quasi-line, for $1 \leq i \leq n$, there exists a partition of $\Gamma\left(C_{i}\right)$ into two cliques: see Fact 1. Also, since $C_{1}, \ldots, C_{n}$ define a tight clique-partition of $V(C)$, the partition is such that $C_{i-p+1}, \ldots, C_{i-1}$ belong to one clique, while $C_{i+1}, \ldots, C_{i+p-1}$ belong to the other. This motivates the following definition:

Definition 11 Let $G$ be a quasi-line graph and $C=\left\{C_{i}, i=1, \ldots, n\right\} \in \mathcal{C}_{G}(n, p)$. For any $i \in\{1, \ldots, n\}$, we denote by $\Gamma^{-}\left(C_{i}\right)$ and $\Gamma^{+}\left(C_{i}\right)$ the classes of a partition of $\Gamma\left(C_{i}\right)$ into two cliques and we assume that $C_{i-p+1} \cup \cdots \cup C_{i-1} \subseteq \Gamma^{-}\left(C_{i}\right)$ and $C_{i+1} \cup \cdots \cup C_{i+p-1} \subseteq \Gamma^{+}\left(C_{i}\right)$.

We are now ready for the main result of this section.

Theorem 6 Let $G$ be a connected fuzzy circular interval graph and $C=\left\{C_{i}, i=\right.$ $1, \ldots, n\} \in \mathcal{C}_{G}(n, p)$. Then, $V(G)=V(C) \cup A(C) \cup B(C) \cup D(C)$.

Proof Let $v \in V(G) \backslash V(C)$ such that $v \in N(C)$. A (non-empty) set $P=\left\{C_{i+q}, q=\right.$ $0, \ldots, l-1\}$ is called a sequence of length $l$ with center $C_{i+\left\lfloor\frac{l-1}{2}\right\rfloor}$. A sequence $P=$ $\left\{C_{i+q}, q=0, \ldots, l-1\right\} \subseteq \Gamma(v, C)$ is maximal with respect to $\Gamma(v, C)$ if $C_{i-1}$ and $C_{i+l} \notin \Gamma(v, C)$.

Claim $2 \Gamma(v, C) \neq \emptyset$.

Let $j \in\{1, \ldots, n\}$ such that $N(v) \cap C_{j} \neq \varnothing$ and $u \in N(v) \cap C_{j}$. We know that without loss of generality $C_{j-1} \subseteq \Gamma^{-}\left(C_{j}\right)$ and $C_{j+1} \subseteq \Gamma^{-}\left(C_{j}\right)$. Observe that $\Gamma\left(C_{j}\right) \subseteq N(u)$. Therefore any partition of $N(u)$ into two cliques $K_{u}^{-}$and $K_{u}^{+}$is such that without loss of generality $C_{j-1} \subseteq K_{u}^{-}$and $C_{j+1} \subseteq K_{u}^{+}$. Since $v \in N(u), v$ belongs either to $K_{u}^{-}$or $K_{u}^{+}$, therefore either $C_{j-1}$ or $C_{j+1}$ belongs to $\Gamma(v, C)$. (end of the claim)

\footnotetext{
1 It is possible to show that, if a fuzzy circular interval graph $G$ contains a tight clique-circulant, then $G$ is connected. Therefore, this assumption is redundant, but we prefer to keep it for simplicity.
} 
Claim 3 If $J \subseteq\{1, \ldots, n\}$ is such that $\bigcup_{i \in J} C_{i}$ is a clique, then $|J| \leq p$.

Let $i \in J$, then, $J \subseteq\{i-p+1, \ldots, i+p-1\}$. But, since $n>2 p, C_{i-h} \cup C_{i-h+p}$ is not a clique, for any $1 \leq h \leq p-1$. Hence, $|J| \leq p$. (end of the claim)

Claim $4|\Gamma(v, C)| \leq 2 p$.

Suppose $|\Gamma(v, C)|>2 p$. As $G$ is quasi-line, $N(v)$ can be partitioned into two disjoint cliques $K_{l}$ and $K_{r}$. If there is no $C_{i}$ such that $C_{i} \cap K_{l} \neq \emptyset$ and $C_{i} \cap K_{r} \neq \emptyset$, then either $K_{l}$ or $K_{r}$ contain at least $p+1$ sets $C_{i}$. But we are then in contradiction with Claim 4.

Hence, let $C_{i}$ be such that $C_{i} \cap K_{l} \neq \emptyset$ and $C_{i} \cap K_{r} \neq \emptyset$, with $K_{l} \cap K_{r}=\emptyset$. Recall that $C_{j} \subset \Gamma\left(C_{i}\right)$ if and only if $|i-j| \leq p-1$. Therefore, since $K_{l}$ and $K_{r}$ are cliques, it follows that $\Gamma(v, C) \subseteq\left\{C_{i-p}, \ldots, C_{i+p}\right\}$; in fact, equality holds, since we are assuming that $|\Gamma(v, C)|>2 p$.

$C_{1}, \ldots, C_{n}$ are the sets of a tight $(n, p)$-clique-partition of $V(C)$, therefore, there is a unique partition of $\Gamma\left(C_{i-1}\right) \cap V(C)$ into two cliques $C_{i-p} \cup \cdots \cup C_{i-2}$ and $C_{i} \cup \cdots \cup C_{i+p-2}$ (cf. Definition 7). Now observe that $\left(\Gamma\left(C_{i-1}\right) \cap V(C)\right) \subset N(v)$. Therefore, without loss of generality, $\left(C_{i} \cup \cdots \cup C_{i+p-2}\right) \subseteq K_{r}$, but, since $C_{i} \nsubseteq K_{r}$, there is a contradiction. (end of the claim)

Claim 5 If $P \subseteq \Gamma(v, C)$ is a sequence which is maximal with respect to $\Gamma(v, C)$, then its length $|P|$ satisfies $2 p-2 \leq|P| \leq 2 p$.

Let $C_{j}$ be the center of $P$. Since $v \in \Gamma\left(C_{j}\right)$, it follows that $v$ belongs either to $\Gamma^{-}\left(C_{j}\right)$ or $\Gamma^{+}\left(C_{j}\right)$. Thus, either $\left\{C_{j-q}, q=1, \ldots, p-1\right\} \subseteq N(v)$ or $\left\{C_{j+q}, q=\right.$ $1, \ldots, p-1\} \subseteq N(v)$. As $C_{j}$ is the center of $P$, we have that $|P| \geq 2(p-1)$. By combining this with the previous claim we get the statement. (end of the claim)

Claim $6 \Gamma(v, C)$ is made of a single sequence of length either $2 p-2$, or $2 p-1$, or $2 p$.

If $P \subseteq \Gamma(v, C)$ is a sequence which is maximal with respect to $\Gamma(v, C)$, then, by Claim 4 and $5,2 p-2 \leq|P| \leq 2 p$. Therefore, in order to prove this claim, it is enough to show that there exists only one maximal sequence in $\Gamma(v, C)$.

Suppose to the contrary that $\Gamma(v, C)$ contains more sequences that are maximal, say $P_{1}, P_{2}, \ldots P_{k}$. Since $P_{i} \cap P_{j}=\emptyset$, for $1 \leq i<j \leq k$, it follows that $2 p \geq|\Gamma(v, C)| \geq$ $\left|P_{1}\right|+\cdots+\left|P_{2}\right| \geq k \cdot(2 p-2)$, where the former inequality follows from Claim 4 and the latter from Claim 5. But then $k=p=2$ and $|\Gamma(v, C)|=\left|P_{1}\right|+\left|P_{2}\right|$, i.e., $\Gamma(v, C)=P_{1} \cup P_{2}$.

Hence, $C \in \mathcal{C}_{G}(n, 2)$. Since $P_{1}$ and $P_{2}$ are not contiguous (else they would not be maximal), we may assume without loss of generality that $\Gamma(v, C)=$ $\left\{C_{1}, C_{2}, C_{i}, C_{i+1}\right\}$ with $3<i<n-1$. Observe that, in particular, $n \geq 6$.

From Lemma 7, there exists a hole $W=\left\{u_{i}, i=1, \ldots, n\right\}$, with $u_{i} \in C_{i}$ for $i=1, \ldots, n$. In particular, if we apply Claim 4 to $\Gamma(v, W)$, it follows that $\Gamma(v, W)=$ $\left\{u_{1}, u_{2}\right\} \cup\left\{u_{i}, u_{i+1}\right\}$. We must now delve into two cases: $n>6$ and $n=6$.

If $n>6$, since $3<i<n-1$, it follows that either $i \geq 5$ or $i \leq$ $n-3$. In the former case, $\left\{v, u_{i}, u_{i+1}, u_{i-1}, u_{i+2}, u_{1}\right\}$ is a net; in the latter case 
$\left\{v, u_{i}, u_{i+1}, u_{i-1}, u_{i+2}, u_{2}\right\}$ is a net. In both cases we are in contradiction with Corollary 1.

The last case is when $n=6$. In this case, $\Gamma(v, W)=\left\{u_{1}, u_{2}\right\} \cup\left\{u_{4}, u_{5}\right\}$. It is possible to show that, in this case, $G[V(W) \cup\{v\}]$ is not a fuzzy circular interval graph (we omit the details, since they are a bit technical and the case where $n \bmod p=0$ is indeed not interesting for the paper). Since each induced subgraph of a fuzzy circular interval graph must be a fuzzy circular interval graph too, we are in contradiction. (end of the claim)

Claim $7 N(v) \subseteq C \cup N(C)$.

First, suppose that $|\Gamma(v, C)|>p$. Suppose to the contrary that there exists $u \in$ $N(v)$ such that $u \notin C \cup N(C)$. Since $\Gamma(v, C)$ is made of a single sequence of length greater than $p$, there exist $x, y \in V(C) \cap N(v)$ such that $x y \notin E(G)$. Then $\{v ; u, x, y\}$ is a claw in $G$, which is a contradiction.

Suppose now that $|\Gamma(v, C)|=p=2$, where the last equality follows Claim 6. In this case, $G$ has a net and this contradicts Corollary 1. (end of the claim)

Finally, since $G$ is connected and, from the last claim, $N(v) \subseteq C \cup N(C)$ when $v \in N(C)$, it follows that $V(G)=V(C) \cup N(C)$. If we combine this with the statement of Claim 6, we get our theorem.

Corollary 3 Let $G$ be a fuzzy circular interval graph. Let $C \in C_{G}(n, p)$ and $v$ a vertex in $N(C)$. If $\alpha(C \cup v)>\alpha(C)$, then $v \in A(C)$ and $n \bmod p=p-1$.

Proof Let $\alpha=\alpha(C)=\left\lfloor\frac{n}{p}\right\rfloor$ by Corollary 2. If $\alpha(C \cup v)>\alpha$, then there exists a stable set $S$ of size $\alpha$ in $G\left[\left\{C_{i}: C_{i} \not \subset N(v)\right\}\right]$. Observe that $\alpha p+p-1 \geq n=\mid\left\{C_{i}\right.$ : $\left.C_{i} \not \subset N(v)\right\}|+|\left\{C_{i}: C_{i} \subset N(v)\right\} \mid \geq(\alpha-1) p+1+2 p-2=(\alpha+1) p-1$. In particular, it follows that $\left|\left\{C_{i}: C_{i} \not \subset N(v)\right\}\right|=2 p-2$, hence $v \in A(C)$.

\subsection{A family of cliques associated with tight clique-circulants}

In this section, we show that a suitable family of cliques can always be associated with clique-circulants in fuzzy circular interval graphs. We need some definitions and a couple of useful lemmas.

Definition 12 Let $G$ be a quasi-line graph and $C=\left\{C_{i}, i=1, \ldots, n\right\} \in \mathcal{C}_{G}(n, p)$. For $i=1, \ldots, n$, we define:

- $A_{i}(C)=\left\{v \in A(C): \Gamma(v, C)=\left\{C_{i-p+2}, \ldots, C_{i+p-1}\right\}\right\}$ (often simply $A_{i}$ );

- $B_{i}(C)=\left\{v \in B(C): \Gamma(v, C)=\left\{C_{i-p+1}, \ldots, C_{i+p-1}\right\}\right\}$ (often simply $B_{i}$ );

- $D_{i}(C)=\left\{v \in D(C): \Gamma(v, C)=\left\{C_{i-p+1}, \ldots, C_{i+p}\right\}\right\} ;$ (often simply $D_{i}$ );

- $D_{i}^{-}(C)=\left\{v \in D_{i}(C): v \in \Gamma\left(D_{i-p+1}(C)\right)\right\}$ (often simply $D_{i}^{-}$);

- $D_{i}^{+}(C)=\left\{v \in D_{i}(C): v \notin \Gamma\left(D_{i-p+1}(C)\right)\right\}$ (often simply $D_{i}^{+}$);

- $L_{i}(C)=C_{i} \cup D_{i}^{-} \cup D_{i-1}^{+}\left(\right.$often simply $\left.L_{i}\right)$.

Lemma 10 Let $G$ be a fuzzy circular interval graph and $C \in C_{G}(n, p)$. Then:

(i) $A_{j} \subset \Gamma^{+}\left(C_{j-p+2}\right), \ldots, \Gamma^{+}\left(C_{j}\right), \Gamma^{-}\left(C_{j+1}\right), \ldots, \Gamma^{-}\left(C_{j+p-1}\right)$; 
(ii) $D_{j} \subset \Gamma^{+}\left(C_{j-p+1}\right), \ldots, \Gamma^{+}\left(C_{j-1}\right), \Gamma^{-}\left(C_{j+2}\right), \ldots, \Gamma^{-}\left(C_{j+p}\right)$;

(iii) $B_{j} \subset \Gamma^{+}\left(C_{j-p+1}\right), \ldots, \Gamma^{+}\left(C_{j-1}\right), \Gamma^{-}\left(C_{j+1}\right), \ldots, \Gamma^{-}\left(C_{j+p-1}\right)$.

Proof (i) Let $k \in\{j-p+2, \ldots, j\}$. By definition of $A_{j}$, if $u \in A_{j}$ then $u \in \Gamma\left(C_{k}\right)$ and $\exists v \in C_{j-p+1}$ such that $(u, v) \notin E(G)$. Since $v \in \Gamma\left(C_{k}\right)$ and, in particular, $v \in \Gamma^{-}\left(C_{k}\right)$ for any $k$, it follows that $u \in \Gamma^{+}\left(C_{k}\right)$. Thus, $A_{j} \subset \Gamma^{+}\left(C_{j-p+2}\right), \ldots, \Gamma^{+}\left(C_{j}\right)$. A similar arguments shows that $A_{j} \subset \Gamma^{-}\left(C_{j+1}\right), \ldots, \Gamma^{-}\left(C_{j+p-1}\right)$.

(ii) Let $l \in\{j-p+1, \ldots, j-1\}$. By definition of $D_{j}$, if $u \in D_{j}$ then $u \in \Gamma\left(C_{l}\right)$; moreover, since $n>2 p, \exists v \in C_{j-p}$ such that $(u, v) \notin E(G)$. Since $v \in \Gamma\left(C_{l}\right)$ and, in particular, $v \in \Gamma^{-}\left(C_{l}\right)$ for any $l$, it follows that $u \in \Gamma^{+}\left(C_{l}\right)$. Thus $D_{j} \subset \Gamma^{+}\left(C_{j-p+1}\right), \ldots, \Gamma^{+}\left(C_{j-1}\right)$. A similar argument shows that $D_{j} \subseteq$ $\Gamma^{-}\left(C_{j+1}\right), \ldots, \Gamma^{-}\left(C_{j+p-1}\right)$. The proof of (iii) goes along the same lines.

Lemma 11 Let $G$ be a fuzzy circular interval graph and $C \in C_{G}(n, p)$. Then, for all $i=1, \ldots, n$, either $A_{i}$ or $D_{i}$ is empty.

Proof Suppose first that $p \geq 3$. Let $u \in D_{i}$. By definition, $C_{i-p+1} \cup \cdots \cup C_{i+p} \subseteq$ $N(u)$, and, since the graph is quasi-line, $N(u)$ can be partitioned into two cliques $K_{l}$ and $K_{r}$. If we apply Lemma 9, first with respect to a vertex in $C_{i}$ and then with respect to a vertex in $C_{i+1}$, we conclude that $C_{i-p+1} \cup \ldots \cup C_{i} \subseteq K_{l}$ and $C_{i+1} \cup \cdots \cup C_{i+p} \subseteq K_{r}$.

Let $v \in A_{i}$. It follows from Lemma 10 that $u$ and $v$ belong to $\Gamma^{+}\left(C_{i-1}\right)$, hence $v \in N(u)$. It follows, either $v \in K_{l}$ or $v \in K_{r}$. But this is not possible since $v$ is neither completely joined to $C_{i-p+1}$ nor to $C_{i+p}$. Hence, either $A_{i}$ or $D_{i}$ is empty.

We now have to deal with the case where $p=2$. Without loss of generality, suppose that that $a \in A_{n}$ and $d \in D_{n}$. First suppose that $(a, d) \notin E$. We claim that, in this case, $N(a) \cap\left(C_{2} \cup C_{n-1}\right)=\emptyset$. Suppose the contrary, e.g. assume that $v \in n(a) \cap C_{2}$, and let $K^{-}(v), K^{+}(v)$ be the cliques of a partition of $N(v)$. From Lemma 9 we may assume that $C_{3} \subseteq K^{+}(v)$, hence $a, d \in K^{-}(v)$, which is a contradiction since $(a, d) \notin E$. From Lemma 7 there exists a hole $W=\left\{v_{i}, i=1, \ldots, n\right\}$, with $v_{i} \in C_{i}$ for $i=1, \ldots, n$. Since $N(a) \cap\left(C_{2} \cup C_{n-1}\right)=\emptyset$, we still have that $a \in A_{n}(W)$ and $d \in D_{n}(W)$. Observe that the set $\left\{v_{n-1}, v_{n}, v_{1}, v_{2}, a, d\right\}$ induce on $G$ a Hajos graph with core $\left\{v_{n}, v_{1}, d\right\}$. But $v_{3}$ is not joined to the core and this contradicts Lemma 3.

Now suppose that $(a, d) \in E .\left\{C_{i}, i=1, \ldots, n\right\}$ is a tight $(n, 2)$-clique-partition of $V(C)$ and therefore, $\Gamma\left(C_{1}\right) \cap V(C)=C_{n} \cup C_{2}$ partitions uniquely into two cliques $\left(C_{n}, C_{2}\right)$. Similarly $\Gamma\left(C_{n}\right) \cap V(C)=C_{n-1} \cup C_{1}$ partitions uniquely into two cliques $\left(C_{n-1}, C_{1}\right) . G$ is quasi-line and thus $N(d)$ also partitions into two cliques $K_{1}, K_{2}$. But $C_{n-1}, C_{n}, C_{1}, C_{2} \subseteq N(d)$. Thus, without loss of generality, $C_{n-1}, C_{n} \subseteq K_{1}$ and $C_{1}, C_{2} \subseteq K_{2}$. But $a \in N(d)$ implies that $a \in K_{1}$ or $a \in K_{2}$ which is impossible since $a \notin \Gamma\left(C_{2}\right)$ and $a \notin \Gamma\left(C_{n-1}\right)$.

Lemma 12 Let $G$ be a fuzzy circular interval graph and $C \in C_{G}(n, p)$. Then $\bigcup_{h=0}^{p-1} L_{i}(C) \bigcup_{h=0}^{p-2} A_{i+h}(C)$ is a clique, for all $i=1, \ldots, n$.

Proof Let $H_{i}(C):=\bigcup_{h=0}^{p-1} L_{i+h}(C) \bigcup_{h=0}^{p-2} A_{i+h}(C)$, for $i=1, \ldots, n$. (i) According to Lemma 11, we delve into two cases: $(j) D_{i}=\emptyset ;(j j) A_{i}=\emptyset$; 
(j) From Lemma 10 and by definition of $D_{i-1}^{+}$, we have $H_{i} \subseteq \Gamma^{+}\left(C_{i}\right)$ and thus $H_{i}$ is a clique.

(jj) By the same argument as above, $H_{i} \backslash D_{i}$ is a clique. It remains to show that $D_{i} \subseteq \Gamma\left(H_{i} \backslash D_{i}\right)$ and that $D_{i}$ is a clique itself. Trivially, $D_{i} \subseteq \Gamma\left(\bigcup_{h=0}^{p-1}\left(C_{i+h}\right)\right)$. Moreover, by looking at $\Gamma^{-}\left(C_{i+p}\right)$, we have that $D_{i} \subseteq \Gamma\left(\bigcup_{h=1}^{p-2}\left(A_{i+h} \cup D_{i+h}\right)\right)$ and that $D_{i}$ is a clique. Finally, by definition of $D_{i+p-1}^{-}, D_{i} \cup D_{i+p-1}^{-}$is a clique. We are thus left to prove that $D_{i} \cup D_{i-1}^{+}$is a clique.

If $p \geq 3$, we know from Lemma 10 that $D_{i-1}^{+}, D_{i} \subseteq \Gamma^{-}\left(C_{i+p-1}\right)$ thus $D_{i} \cup D_{i-1}^{+}$ is a clique and the result follows.

Now we deal with $p=2$. By definition of $D_{i}^{-}, D_{i}^{-} \cup D_{i-1}^{+}$is a clique. It is left to prove that $D_{i}^{+} \cup D_{i-1}^{+}$is a clique. Suppose the contrary, then there exist $a \in$ $D_{i}^{+}, b \in D_{i-1}^{+}$and $c \in D_{i-2}$ such that $(a, b),(b, c) \notin E$. But $c \in \Gamma^{-}\left(C_{i}\right)$, thus $a \in \Gamma^{-}\left(C_{i}\right)$ also and $(a, c) \in E$. We know from Lemma 7 that there exists a hole $W=\left\{u_{i}, i=1, \ldots, n\right\}$, with $u_{i} \in C_{i}$ for $i=1, \ldots, n$. Observe that $a, b, c \in D(W)$. If $n>5$, then $\left\{u_{i}, u_{i+1}, u_{i+2}, a, b, c\right\}$ induces a Hajos graph in $G$ and $u_{i-2}$ is not connected to the core $\left\{a, u_{i}, u_{i+1}\right\}$ which contradicts Lemma 3 .

The last case is when $n=5$. Case analysis shows that, in this case, if $D_{i}^{+} \cup D_{i-1}^{+}$ is not a clique, then $G$ is not a fuzzy circular interval graph. We omit the details.

The above lemma is crucial for characterizing the family of cliques defined in the next Theorem.

Theorem 7 Let $G$ be a fuzzy circular interval graph and $C \subseteq G$ a tight $(n, p)$-cliquecirculant. There exists a family $\mathcal{F}$ of $n$ cliques of $G$ such that:

(a) Every vertex in $V(C) \cup D(C)$ is covered by p distinct cliques of $\mathcal{F}$;

(b) every vertex in $A(C)$ is covered by $p-1$ distinct cliques of $\mathcal{F}$.

Proof From Lemma 12 we have that $H_{i}:=\bigcup_{h=0}^{p-1} L_{i+h}(C) \bigcup_{h=0}^{p-2} A_{i+h}(C)$ is a clique, for $i=1, \ldots, n$. Then family $\mathcal{F}=\left\{H_{i}, i=1, \ldots, n\right\}$ satisfies the above conditions.

\subsection{The relationship between clique-circulants and tight clique-circulants}

Now that we have introduced the class of tight clique-circulants and that we studied its properties, we are ready to relate it to the original class of clique-circulants. In fact, tight clique-circulants allow to build more structural "representations" of cliquecirculants and we will often switch to the tight clique-circulant representation in our constructive proofs.

Lemma 13 If $G$ is an $(n, p)$-clique-circulant, then there exists a tight $(n, p)$-cliquecirculant $C$ such that $V(G)=V(C) \cup D(C)$.

Proof Let $\left\{Q_{i}, i=1, \ldots, n\right\}$ be an $(n, p)$-clique-partition of $V(G)$ and $W=$ $\left\{v_{1}, \ldots, v_{n}\right\}$ an $(n, p)$-circulant, with $v_{i} \in Q_{i}$. The tight $(n, p)$-clique-circulant $C=$ $\left\{C_{1}, \ldots, C_{n}\right\}$ is built by the following algorithm. First, set $C_{i}=\left\{v_{i}\right\}$, for $i=1, \ldots, n$. 
Then, as long as there exists a vertex $v \in Q_{i} \backslash C_{i}$ such that $v \notin \Gamma\left(C_{i-p}\right) \cup \Gamma\left(C_{i+p}\right)$ for some $i \in\{1, \ldots, n\}$ - add $v$ to $C_{i}$, i.e. set $C_{i}=C_{i} \cup\{v\}$.

We claim that the graph $C=\left\{C_{1}, \ldots, C_{n}\right\}$ defined in this way is a tight $(n, p)$ clique-circulant. The proof is by induction. Our statement is true when $C_{i}=\left\{v_{i}\right\}$ from Lemma 8.

Now suppose that $C=\left\{C_{1}, \ldots, C_{n}\right\}$ is a tight $(n, p)$-clique-circulant $C$ and we add a vertex $v \in Q_{i}$, with $v \notin \Gamma\left(C_{i-p}\right) \cup \Gamma\left(C_{i+p}\right)$, to $C_{i}$. We have to show that also $C^{\prime}=\left\{C_{1}, \ldots, C_{i-1}, C_{i} \cup\{v\}, C_{i+1}, \ldots, C_{n}\right\}$ is a tight $(n, p)$-clique-circulant. Since the sets $Q_{1}, \ldots, Q_{n}$ define a an $(n, p)$-clique-partition of $V(G)$ and $C_{j} \subseteq Q_{j}$ for each $j$, it follows that $C_{i-p+1} \cup \cdots \cup C_{i-1} \cup C_{i+1} \cup \ldots \cup C_{i+p-1} \subseteq \Gamma\left(Q_{i}\right) \subseteq N(v)$ and therefore, since $v \notin \Gamma\left(C_{i-p}\right) \cup \Gamma\left(C_{i+p}\right), v \in B_{i}(C)$. Hence, $\left(C_{i} \cup\{v\}\right) \subseteq \Gamma\left(C_{j}\right)$ if and only if $|j-i| \leq p-1, j \neq i$.

It follows that, in order to show that $C^{\prime}$ is a tight $(n, p)$-clique-circulant, it remains to show that in $G\left[V\left(C^{\prime}\right)\right]$ there is a unique partition of $\Gamma\left(C_{j}\right)$ into two cliques, with $C_{j}=C_{1}, \ldots, C_{i-1}, C_{i} \cup\{v\}, C_{i+1}, \ldots, C_{n}$. Clearly, it is enough to deal with sets $C_{j}$ such that $v \in \Gamma\left(C_{j}\right)$, i.e. $C_{i-p+1}, \ldots, C_{i-1}, C_{i+1}, \ldots, C_{i+p-1}$. Observe that for such sets exactly one between $C_{i-p}$ and $C_{i+p}$ belongs to $\Gamma\left(C_{j}\right)$ : say it is $C_{i-p}$. By induction, in $G[V(C)]$ the partition of $\Gamma\left(C_{j}\right)$ into two cliques is unique and, in particular, $C_{i-p}$ belongs to one clique. Then, since $v \notin \Gamma\left(C_{i-p}\right)$, the partition of $\Gamma\left(C_{j}\right)$ remains unique also in $G\left[V\left(C^{\prime}\right)\right]$.

Finally, let $v$ be a vertex that at the end of the algorithm belongs to $Q_{i} \backslash C_{i}$, for some $i \in\{1, \ldots, n\}$. It follows that either $C_{i-p} \subset N(v)$ or $C_{i+p} \subset N(v)$. Also, since the sets $Q_{1}, \ldots, Q_{n}$ define an $(n, p)$-clique-partition of $V(G)$ and $C_{j} \subseteq Q_{j}$ for each $j$, it follows that $C_{i-p+1} \cup \cdots \cup C_{i+p-1} \subseteq \Gamma\left(Q_{i}\right) \subseteq N(v)$. Therefore, $v$ is a vertex of type $D$.

The graph $G$ in Fig. 1 is a $(5,2)$-clique-circulant. In fact, it is easy to see that there exists a tight $(5,2)$-clique-circulant $C$ such that $V(G)=V(C) \cup D(C)$. Lemma 13 can be "reverted". We have in fact the following:

Lemma 14 Let $G$ be a fuzzy circular interval graph and $C \subseteq G$ a tight $(n, p)$-cliquecirculant. The graph $G[V(C) \cup D(C)]$ is an $(n, p)$-clique-circulant.

Proof $L_{1}, \ldots, L_{n}$ are the classes of an $(n, p)$-clique-partition of the vertices of $V(C) \cup D(C)$. In fact, by definition, $L_{1}, \ldots, L_{n}$ partition $V(C) \cup D(C)$ into $n$ classes. Moreover, $\Gamma\left(L_{i}\right) \supseteq\left(L_{i-p+1} \cup \cdots \cup L_{i+p-1}\right)$, for $i=1, \ldots, n$, since $\bigcup_{h=0}^{p-1} L_{i+h}$ is a clique (see Lemma 12).

We must then show that there exists an $(n, p)$-circulant $W=\left\{v_{i}, i=1, \ldots, n\right\}$, with $v_{i} \in L_{i}$ for $i=1, \ldots, n$. This follows from Lemma 7, since $C_{i} \subseteq L_{i}$, for $i=1, \ldots, n$.

4.4 The key ingredient to the characterization of the ranks facets: the "invariance" of clique-circulants

In this section we show that clique-circulants are invariant with respect to the addition of vertices not increasing the stability number and give therefore a proof of Theorem 5 . This proof builds upon a few lemmas. 
Lemma 15 Let $G$ be a fuzzy circular interval graph, $C \in C_{G}(n, p)$ such that $d=$ $\operatorname{gcd}(n, p)>1$. There exists $C^{\prime} \in C_{G}(n / d, p / d)$ such that $V(C) \cup D(C) \subseteq V\left(C^{\prime}\right) \cup$ $D\left(C^{\prime}\right)$ and $A\left(C^{\prime}\right)=\emptyset$.

Proof Let $C=\left\{C_{i}, i=1, \ldots, n\right\}, d=\operatorname{gcd}(n, p)>1, n^{\prime}=n / d, p^{\prime}=p / d$ and $G^{\prime}=G\left[\bigcup_{q=1, \ldots, n^{\prime}} C_{q p}\right]$. We know from Lemma 7 that there exists an $(n, p)$-circulant $W=\left\{v_{1}, \ldots, v_{n}\right\}$ such that $v_{i} \in C_{i}$ for $i=1, \ldots, n$. Observe that $W^{\prime}=\left\{v_{q p}, q=\right.$ $\left.1, \ldots, n^{\prime}\right\}$ is an $\left(n^{\prime}, p^{\prime}\right)$-circulant and that the sets $\left\{C_{q p}, q=1, \ldots, n^{\prime}\right\}$ define an $\left(n^{\prime}, p^{\prime}\right)$-clique-partition of $V\left(G^{\prime}\right)$.

Therefore, $G^{\prime}$ is an $\left(n^{\prime}, p^{\prime}\right)$-clique-circulant and, by Lemma 13 to $G^{\prime}$, there exists a tight $\left(n^{\prime}, p^{\prime}\right)$-clique-circulant $C^{\prime}=\left\{C_{p}^{\prime}, \ldots, C_{n^{\prime} p}^{\prime}\right\}$ such that, for $q=1, \ldots, n^{\prime}$, $C_{q p}^{\prime} \subseteq C_{q p}$ and each vertex in $C_{q p} \backslash C_{q p}^{\prime}$ belongs to $D\left(C^{\prime}\right)$.

Consider now a vertex $v \in C_{i}$ with $i \notin\left\{q p, q=1, \ldots, n^{\prime}\right\}$. In this case, $\mid\{q p, q=$ $\left.1, \ldots, n^{\prime}\right\} \cap\{i-p+1, \ldots, i-1\} \mid=p^{\prime}$ and $\mid\left\{q p, q=1, \ldots, n^{\prime}\right\} \cap\{i+1, \ldots, i+$ $p-1\} \mid=p^{\prime}$. Then $\left|\Gamma\left(v, C^{\prime}\right)\right|=2 p^{\prime}$. Thus $v \in D\left(C^{\prime}\right)$.

Analogously, if $v \in D(C)$, then $\left|\Gamma\left(v, C^{\prime}\right)\right| \geq\left\lfloor\frac{|\Gamma(v, C)|}{d}\right\rfloor \geq\left\lfloor\frac{2 p}{d}\right\rfloor=2 p^{\prime}$ and so $v \in D\left(C^{\prime}\right)$. Hence $V(C) \cup D(C) \subseteq V\left(C^{\prime}\right) \cup D\left(C^{\prime}\right)$. Thus $v \in D\left(C^{\prime}\right)$.

Finally, consider a vertex $v \notin V(C) \cup D(C)$. We know that $|\Gamma(v, C)| \geq 2 p-2$. On the other hand, $\left|\Gamma\left(v, C^{\prime}\right)\right| \geq\left\lfloor\frac{|\Gamma(v, C)|}{d}\right\rfloor \geq\left\lfloor\frac{2 p-2}{d}\right\rfloor \geq 2 p^{\prime}-1$ if $d>1$. Thus $v \in B\left(C^{\prime}\right) \cup D\left(C^{\prime}\right)$.

Lemma 16 Let $G$ be a fuzzy circular interval graph, $C \in C_{G}(n, p)$ and $b \in B(C)$. There exists $\bar{C} \in C_{G}(n, p)$ such that $\{b\} \cup V(C) \cup D(C) \subseteq V(\bar{C}) \cup D(\bar{C})$.

Proof Without loss of generality let $b \in B_{n}(C) . L_{1}, \ldots, L_{n}$ are the classes of an $(n, p)$-clique-partition of the vertices of $V(C) \cup D(C)$, where $L_{i}=C_{i} \cup D_{i}^{-}(C) \cup$ $D_{i-1}^{+}(C), i=1, \ldots, n$. In fact, by definition, $L_{1}, \ldots, L_{n}$ partition $V(C) \cup D(C)$ into $n$ classes. Moreover, $\Gamma\left(L_{i}\right) \supseteq\left(L_{i-p+1} \cup \ldots \cup L_{i+p-1}\right)$, for $i=1, \ldots, n$, since $\bigcup_{h=0}^{p-1} L_{i+h}$ is a clique (see Lemma 12 ).

Observe that we obtain a possibly different $(n, p)$-clique-partition $L_{1}^{\prime}, \ldots, L_{n}^{\prime}$ of $V(C) \cup D(C)$ if we let $C_{i}^{\prime}=C_{n-i}$, for $i=1, \ldots n$, and, as usual, $L_{i}^{\prime}=C_{i}^{\prime} \cup D_{i}^{-}\left(C^{\prime}\right) \cup$ $D_{i-1}^{+}\left(C^{\prime}\right)$. Also observe that, by definition, $b \in B_{n}\left(C^{\prime}\right)$.

We will show that either $L_{n-p+1} \cup \cdots \cup L_{p-1} \subseteq N(b)$ or $L_{n-p+1}^{\prime} \cup \cdots \cup$ $L_{p-1}^{\prime} \subseteq N(b)$. Trivially, this implies that either $\left\{L_{1}, \ldots, L_{n-1},\left(L_{n} \cup\{b\}\right)\right\}$ or $\left\{L_{1}^{\prime}, \ldots, L_{n-1}^{\prime},\left(L_{n}^{\prime} \cup\{b\}\right)\right\}$ defines an $(n, p)$-clique-partition of $\{v\} \cup V(C) \cup D(C)$. The result will then follow since by Lemma 7 there exists an $(n, p)$-circulant $W=\left\{w_{1}, \ldots, w_{n}\right\}$ with $w_{i} \subseteq C_{i}$ and hence, since $C_{i} \subseteq L_{i}$, we can apply Lemma 13 .

By definition, $L_{n-p+1} \cup \cdots \cup L_{p-1}=C_{n-p+1} \cup \cdots \cup C_{p-1} \cup D_{n-p+1}(C) \cup$ $\cdots \cup D_{p-2}(C) \cup D_{n-p}^{+}(C) \cup D_{p-1}^{-}(C)$. Since $b \in B_{n}(C)$, it follows that $N(b) \supseteq C_{n-p+1} \cup \ldots \cup C_{p-1}$. Also, by Lemma 10, $D_{n-p+1}(C) \cup\{b\} \subseteq$ $\Gamma^{-}\left(C_{1}\right), \ldots, D_{n-1}(C) \cup\{b\} \subseteq \Gamma^{-}\left(C_{p-1}\right), D_{n}(C) \cup\{b\} \subseteq \Gamma^{-}\left(C_{p-1}\right)$ and $D_{1}(C) \cup\{b\} \subseteq \Gamma^{+}\left(C_{n-p+2}\right), \ldots, D_{p-2}(C) \cup\{b\} \subseteq \Gamma^{+}\left(C_{n-1}\right)$ : therefore, $N(b) \supseteq$ $D_{n-p+1}(C) \cup \ldots \cup D_{p-2}(C)$.

Moreover $D_{n-p}^{+}(C) \cup\{b\} \subseteq \Gamma^{+}\left(C_{n-p+1}\right)$. In fact, $b \in \Gamma^{+}\left(C_{n-p+1}\right)$ from Lemma 10. As for $D_{n-p}^{+}(C)$, by definition, for each $v \in D_{n-p}^{+}(C)$ there exists a 

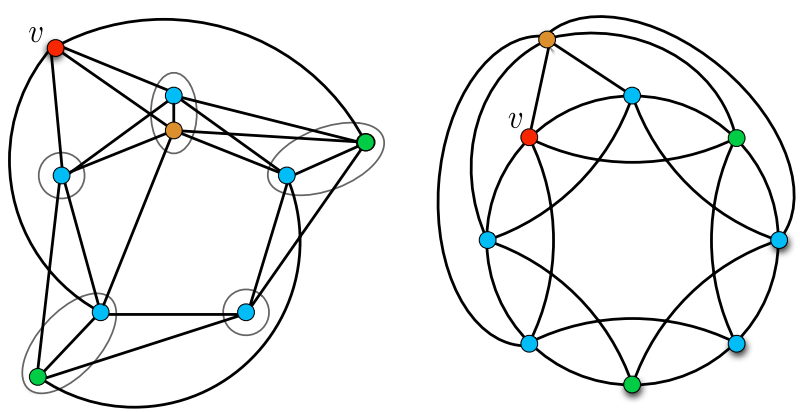

Fig. 4 Example of A-extension when $\operatorname{gcd}(\mathrm{n}, \mathrm{p})=1$

vertex $w \in D_{n-2 p+1}(C)$ not adjacent to $v$. Since $D_{n-2 p+1}(C) \subseteq \Gamma^{-}\left(C_{n-p+1}\right)$, it follows that $D_{n-p}^{+}(C) \subseteq \Gamma^{+}\left(C_{n-p+1}\right)$. Therefore $N(b) \supseteq D_{n-p}^{+}(C)$.

By the same arguments, it follows that $L_{n-p+1}^{\prime} \cup \cdots \cup L_{p-1}^{\prime}=C_{n-p+1}^{\prime} \cup \cdots \cup$ $C_{p-1}^{\prime} \cup D_{n-p+1}\left(C^{\prime}\right) \cup \cdots \cup D_{p-2}\left(C^{\prime}\right) \cup D_{n-p}^{+}\left(C^{\prime}\right) \cup D_{p-1}^{-}\left(C^{\prime}\right)$ and that $N(b) \supseteq$ $C_{n-p+1}^{\prime} \cup \cdots \cup C_{p-1}^{\prime} \cup D_{n-p+1}\left(C^{\prime}\right) \cup \cdots \cup D_{p-2}\left(C^{\prime}\right) \cup D_{n-p}^{+}\left(C^{\prime}\right)$.

Therefore, it remains to show that either $N(b) \supseteq D_{p-1}^{-}(C)$ or $N(b) \supseteq D_{p-1}^{-}\left(C^{\prime}\right)$. Observe that $D_{p-1}\left(C^{\prime}\right)=D_{n-p}(C)$. By Lemma 10, $D_{p-1}(C) \subseteq \Gamma^{-}\left(C_{n}\right)$ while $D_{n-p}(C) \subseteq \Gamma^{+}\left(C_{n}\right)$. Since $b \in \Gamma\left(C_{n}\right)$ it follows that either $N(b) \supseteq D_{p-1}(C) \supseteq$ $D_{p-1}^{-}(C)$ or $N(b) \supseteq D_{n-p}(C)=D_{p-1}\left(C^{\prime}\right) \supseteq D_{p-1}^{-}\left(C^{\prime}\right)$.

Lemma 17 Let $G$ be a fuzzy circular interval graph, $C \in C_{G}(n, p)$ with $\operatorname{gcd}(n, p)=$ 1 and $v_{0} \in A(C)$. If $\alpha(\{v\} \cup C \cup D(C))=\alpha(C)$, then there exists $\bar{C} \in C_{G}\left(n^{\prime}, p^{\prime}\right)$, with $\left\lfloor\frac{n^{\prime}}{p^{\prime}}\right\rfloor=\left\lfloor\frac{n}{p}\right\rfloor$, such that $v_{0} \in V(\bar{C})$ and $V(C) \cup D(C) \subseteq V(\bar{C}) \cup D(\bar{C})$.

Lemma 17 might seem quite surprising since we move from a tight $(n, p)$-cliquecirculant to a tight $\left(n^{\prime}, p^{\prime}\right)$-clique-circulant. In fact, the constructive proof this Lemma is a bit technical and we prefer to defer it to Appendix B. An example can be found in Fig. 4 (observe that this is again Fig. 2) where there is a tight $(5,2)$-clique-circulant and a vertex $v$ of type A, that does not increase the stability number. Applying the A-extension algorithm of Appendix B, we realize that this graph is nothing but a $(8,3)$-circulant plus a vertex of type $D$ (Fig. 4 ).

We are now ready to recall and prove Theorem 5.

Theorem 5. Let $G(V, E)$ be a fuzzy circular interval graph, $Q \subset V$ such that $G[Q]$ is an $(n, p)$-clique-circulant and $v \notin Q$ such that $\alpha(Q \cup\{v\})=\alpha(Q)$. Then $G[Q \cup\{v\}]$ is an $\left(n^{\prime}, p^{\prime}\right)$-clique-circulant, with $\left\lfloor\frac{n^{\prime}}{p^{\prime}}\right\rfloor=\left\lfloor\frac{n}{p}\right\rfloor$.

Proof $G[Q]$ is an $(n, p)$-clique-circulant. Then, from Lemma 13, there exists a tight $(n, p)$-clique-circulant $C$ such that $Q \subseteq V(C) \cup D(C)$. Observe that from Lemma 15 we may assume, without loss of generality, that $\operatorname{gcd}(n, p)=1$.

From Theorem 6, $V(G)=V(C) \cup A(C) \cup B(C) \cup D(C)$. Then the theorem follows from Lemma 14 if $v \in V(C) \cup D(C)$. Else either $v \in B(C)$ or $v \in A(C)$.

In the former case, from Lemma 16 there exists $\bar{C} \in C_{G}(n, p)$ such that $V(\bar{C}) \cup$ $D(\bar{C}) \supseteq\{b\} \cup V(C) \cup D(C) \supseteq\{b\} \cup Q$. The theorem then follows from Lemma 14 applied to $\bar{C}$. 
If $v \in A(C)$, since $\operatorname{gcd}(n, p)=1$, we apply Lemma 17 and the theorem follows again from Lemma 14.

\section{The rank facets of fuzzy circular interval graphs and a conjecture on all facets}

We are now ready to prove that main result of this paper. In fact, Theorem 5 allows for a combinatorial characterization of all the rank facets of the stable set polytope of a fuzzy circular interval graph.

Theorem 8 Let $G=(V, E)$ be a fuzzy circular interval graph. An inequality $\sum_{v \in Q} x_{v} \leq \alpha(Q)$, with $\alpha(Q) \geq 2$, is a facet of $S T A B(G)$ if and only if $G[Q]$ is an $\alpha$-maximal $(n, p)$-clique-circulant, with $n \bmod p \neq 0$.

Proof Necessity. Since $\sum_{v \in Q} x_{v} \leq \alpha(Q)$ is facet inducing for $S T A B(G)$, it follows from Lemma 5 that $G[Q]$ is $\alpha$-maximal. From Corollary 4, there exists $W \subseteq Q$ that is an $(\alpha(Q) p+1, p)$-circulant, $p \geq 2 . W$ is a clique-circulant (Lemma 6). Then, by induction, from Theorem $5, G[Q]$ is an $(n, p)$-clique-circulant, for some $n$ and $p$, with $\left\lfloor\frac{n}{p}\right\rfloor=\alpha(Q)$. Finally, $n \bmod p \neq 0$, else the inequality $\sum_{j \in Q} x_{j} \leq\left\lfloor\frac{n}{p}\right\rfloor$ is not a facet of $S T A B(G[Q])$ (Theorem 4) and therefore not a facet of $S T A B(G)$ (Lemma 5).

Sufficiency. Since $n \bmod p \neq 0, \sum_{j \in Q} x_{j} \leq \alpha(Q)$ is a facet of $\operatorname{STAB}(G[Q])$ from Theorem 4. Since $G[Q]$ is $\alpha$-maximal, the statement follows from Lemma 5 .

We can therefore state the following theorem for fuzzy circular interval graphs.

Theorem 9 Let $G=(V, E)$ be a fuzzy circular interval graph. An inequality $\sum_{v \in V\left(G^{\prime}\right)} x_{v} \leq \alpha\left(G^{\prime}\right)$ is a facet of $S T A B(G)$ if and only if $G^{\prime}$ is

- a maximal clique or

- an $\alpha$-maximal $(n, p)$-clique-circulant with $n \bmod p \neq 0$.

The next corollary follows directly from Theorem 7, Lemma 13 and Theorem 1.

Corollary 4 Let $G=(V, E)$ be a fuzzy circular interval graph and $C a(n, p)$ clique-circulant. Let $r=n \bmod p$. The following inequality is valid for $S T A B(G)$ :

$$
(p-r) \cdot \sum_{v \in V(C)} x_{v}+(p-r-1) \cdot \sum_{v \in N(C)} x_{v} \leq(p-r) \cdot\left\lfloor\frac{n}{p}\right\rfloor .
$$

Proof We know from Lemma 13 that there exists a tight $(n, p)$-clique-circulant $C^{\prime}$ such that $V(C)=V\left(C^{\prime}\right) \cup\left(D\left(C^{\prime}\right) \cap V(C)\right)$. We know from Theorem 6 that $V(G)=$ $V\left(C^{\prime}\right) \cup A\left(C^{\prime}\right) \cup B\left(C^{\prime}\right) \cup D\left(C^{\prime}\right)$. Hence $N(C) \subseteq A\left(C^{\prime}\right) \cup B\left(C^{\prime}\right) \cup D\left(C^{\prime}\right)$. By iteratively applying Lemma 16 , we can find a tight $(n, p)$-clique-circulant $\bar{C}$ such that $B(\bar{C})=\emptyset, V(C) \subseteq V(\bar{C}) \cup D(\bar{C})$ and $N(C) \subseteq A(\bar{C}) \cup D(\bar{C})$. Now applying Theorem 7 and Theorem 1 shows that the inequality $(p-r) \cdot \sum_{v \in V(\bar{C}) \cup D(\bar{C})} x_{v}+(p-$ $r-1) \cdot \sum_{v \in A(\bar{C})} x_{v} \leq(p-r) \cdot\left\lfloor\frac{n}{p}\right\rfloor$ is valid for $S T A B(G)$. But this later inequalities dominates inequality (3). 
Fig. $52 \sum_{v \in \bullet} x_{v}+\sum_{v \in o} x_{v} \leq 4$

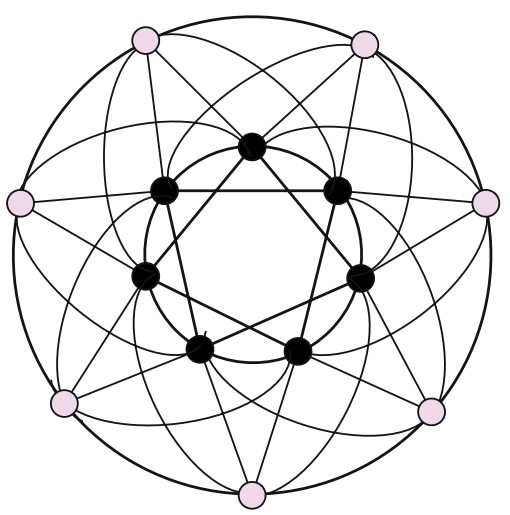

Observe that the inequality (3) — that we call the clique family inequality associated with $C$-is a rank inequality if either $N(C)=\emptyset$ or $r=p-1$ : in fact, in this case, it reads $\sum_{v \in V(C)} x_{v} \leq\left\lfloor\frac{n}{p}\right\rfloor$.

Some authors $[9,11,16]$ provided non-rank facets for $S T A B(G)$, when $G$ is a fuzzy circular interval graph. Recall that quasi-line graphs that are not fuzzy circular interval graph have only rank facets (see Sect. 1). It is routine to check that all these inequalities can be expressed as inequalities (3). For instance, if we consider the graph $G$ in Fig. 5, the inequality $2 \sum_{v \in \bullet} x_{v}+\sum_{v \in o} x_{v} \leq 4$-which is facet-inducing for $\operatorname{STAB}(G)$ [9] - can be equivalently expressed as $2 \sum_{v \in V(C)} x_{v}+\sum_{v \in N(C)} x_{v} \leq 4$, where $C$ is the $(7,3)$-circulant induced by the black vertices.

We should also observe that all the non-rank facets we know are such that the induced clique-circulant in inequality (3) is maximal.

Definition 13 Let $G=(V, E)$ be a fuzzy circular interval graph. An induced $(n, p)$ clique-circulant $C$ of $G$ is maximal if for each vertex $v$ such that $v \in N(C), C \cup v$ is not an $(n, p)$-clique-circulant.

Note that a $(n, p)$-clique-circulant that is $\alpha$-maximal is also maximal but the converse is not necessarily true. In the case of the graphs from Fig. 2 for instance, it is not possible to find a $(5,2)$-clique-circulant including the vertex $v$.

Our results thus suggest, for fuzzy circular interval graphs, a conjecture strengthening the Ben Rebea theorem. As we have just shown, this conjecture holds for rank facets.

Conjecture 2 Each facet of the stable set polytope of a fuzzy circular interval graph is either:

- a sign inequality,

- a maximal clique inequality or

- a clique family inequality (3) associated with a maximal $(n, p)$-clique-circulant $C$ such that $n \bmod p \neq 0$.

Acknowledgments The authors wish to thank an anonymous referee for her/his very helpful comments and suggestions. 


\section{A Proof of Lemma 4}

There are several standard techniques for producing facets of the SSP from "smaller" ones. When dealing with claw-free graphs, (i) sequential lifting (cf. definition in Sect. 3) and (ii) complete join seem so far to be the most interesting.

(ii) Let $G_{1}$ and $G_{2}$ be graphs. We denote by $G_{1}+G_{2}$ the (disjoint) union of $G_{1}$ and $G_{2}$ together with additional edges that join each vertex of $G_{1}$ to each vertex of $G_{2}$. Chvátal [4] proved that a complete description of $S T A B\left(G_{1}+G_{2}\right)$ can be easily derived from one of $S T A B\left(G_{1}\right)$ and $S T A B\left(G_{2}\right)$. In particular, if $\sum_{j \in V\left(G_{1}\right)} a_{j} x_{j} \leq b_{1}$ is a facet of $S T A B\left(G_{1}\right)$ and $\sum_{j \in V\left(G_{2}\right)} a_{j} x_{j} \leq b_{2}$ is a facet of $S T A B\left(G_{2}\right)$ then $b_{2} \sum_{j \in V\left(G_{1}\right)} a_{j} x_{j}+b_{1} \sum_{j \in V\left(G_{2}\right)} a_{j} x_{j} \leq b_{1} b_{2}$ is a facet of $S T A B\left(G_{1}+G_{2}\right)$ and is called the complete join of the two inequalities.

It is therefore of interest to characterize the facets which do not arise from sequential lifting and/or complete join. Galluccio and Sassano [8] have exploited this approach for the class of rank facets of a claw-free graph $G(V, E)$. They define a rank facet $\sum_{j \in V} x_{j} \leq \alpha(G)$ to be rank-minimal if, for any $T \subset V$, the inequality $\sum_{j \in T} x_{j} \leq$ $\alpha(G)$ is not a facet of $S T A B(G[T])$. Their first result shows how to construct rank facets from rank-minimal ones in claw-free graphs.

Theorem 10 [8] Let $G=(V, E)$ be a claw-free graph. Every rank facet can be constructed from a rank-minimal one by means of sequential lifting and/or complete join.

This theorem can be sharpened when dealing with quasi-line graphs. First, observe that there do exist non-minimal rank facets of the SSP of a claw-free graph which cannot be produced from the sequential lifting of smaller ones: an example is given by the inequality $\sum_{v \in V(G)} x_{j} \leq 2$ for the graph $G=C_{5}+C_{5}$. This is not the case for quasi-line graphs because of the following lemma.

Lemma 18 [16] Let a quasi-line graph $G$ be the complete join of graphs $G_{1}$ and $G_{2}$. Then $G$ is perfect.

According to this lemma, if $\sum_{j \in V} x_{j} \leq \alpha(V)$ is facet-inducing for some quasi-line graph $G(V, E)$ that is the complete join of graphs $G_{1}$ and $G_{2}$, then $G$ is a clique.

Now, let $K$ be a clique for some graph $G$. The clique inequality $\sum_{j \in K} x_{j} \leq 1$ can be produced by sequential lifting from the inequality $x_{v} \leq 1$, where $v$ is any vertex of $K$. We may therefore sharpen Theorem 10 as follows:

Corollary 5 Let $G(V, E)$ be a quasi-line graph. Every rank facet can be constructed from a rank-minimal one by means of sequential lifting.

The main result by Galluccio and Sassano is the characterization of rank-minimal facets of claw-free grahs.

Definition 14 A graph $G$ is hypomatchable if, for each $v \in V(G)$, there exists a perfect matching in $G[V \backslash\{v\}]$.

Theorem 11 [8] Let $G=(V, E)$ be a claw-free graph such that $\sum_{v \in V} x_{v} \leq \alpha(G)$ is facet inducing and rank-minimal. Then one of the following statements holds: 
(i) $G$ is a singleton;

(ii) $G$ is the line graph of a minimal 2-connected hypomatchable graph $H$ (i.e. $H$ is such that $H \backslash e$ is no more hypomatchable for any $e \in E(H))$;

(iii) $G$ is $a(\alpha \omega+1, \omega)$-circulant, $\omega \geq 3$.

This theorem can be sharpened too, but we now consider the class of fuzzy circular interval graphs, where we have the following lemma.

Lemma 19 Let $G(V, E)$ be a fuzzy circular interval graph. If $H \subseteq G$ is the line graph of a minimal 2-connected hypomatchable graph, then $H$ is an odd hole.

Proof Let $G$ be the line graph of a minimal 2-connected hypomatchable graph. It is straightforward to check that, if $G$ is not an odd hole, then $G$ must contain a net. This is not possible for fuzzy circular interval graphs (Corollary 1).

Lemma 4 is therefore a corollary of Theorem 11, Lemma 19 and Lemma 5.

\section{B Proof of Lemma 17}

Let us first describe the intuition behind this Lemma. Take the example of Fig. 2. We need to define a clique-circulant including the extra vertex $v$ from a tight $(5,2)$ clique-circulant. By simple enumeration, one can quickly realize that there is no hope to find a $(5,2)$-clique-criculant. We are therefore looking for a new circulant structure. Now the corresponding clique-circulant contains, by definition, a circulant. The idea of the algorithm is therefore to look for a new circulant including the new vertex $v$. Suppose this circulant is a $\left(n^{\prime}, p^{\prime}\right)$-circulant and $v$ is numbered 0 in this circulant. the vertex numbered $p^{\prime}$ is not adjacent to $v$ so it can neither be in $C_{1}, \ldots, C_{p-2}$ nor in $C_{p-1}$. We should therefore look for this potential vertex in $C_{p}, C_{p+1}$ or higher. We bet that we can find this vertex in $C_{p}$. We mark the potential candidates $Q$ (those not complete to $v$ ) and we iterate the reasoning with those potential candidates (i.e. we look at the vertices in $C_{2 p}$ not complete to $Q$, mark them and go on). As soon as $v$ is a candidate for being the successor of the current candidates $Q$ (note that this can only happen when we are dealing with candidates $Q \subset C_{n-p+1}$ ), we can show that we have detected a new circulant structure and the construction provides a new tight clique-circulant with the required properties (cf. Claim 11). If at some point the set of candidates $Q$ is empty, we cannot detect a circulant but we can slightly change the original tight $(n, p)$-clique-circulant so that $v$ becomes of type $\mathrm{B}$ and the results is a corollary of Lemma 16 (cf. Claim 9). In both situations, the conclusion of Lemma 17 are satisfied. Note that we can visit many times the set $C_{i}$ s to search for candidates and thus we keep track of the number of times we "wind" around $C$ by incrementing $m$. We denote by $C_{i}^{k}$ the vertices marked in set $C_{i}$ in round $k$ and by $R_{i}^{k}$ the vertices in $C_{i}$ not yet marked after round $k$.

This algorithm is described formally in Algorithm 1.

In the example of Fig. 2, we have detected a $(8,3)$-circulant.

We denote by $\left(n_{0}, p_{0}\right)$ the unique pair of integers such that $n_{0} p-p_{0} n=1$ and $n_{0}<n($ recall that $\operatorname{gcd}(n, p)=1)$. 


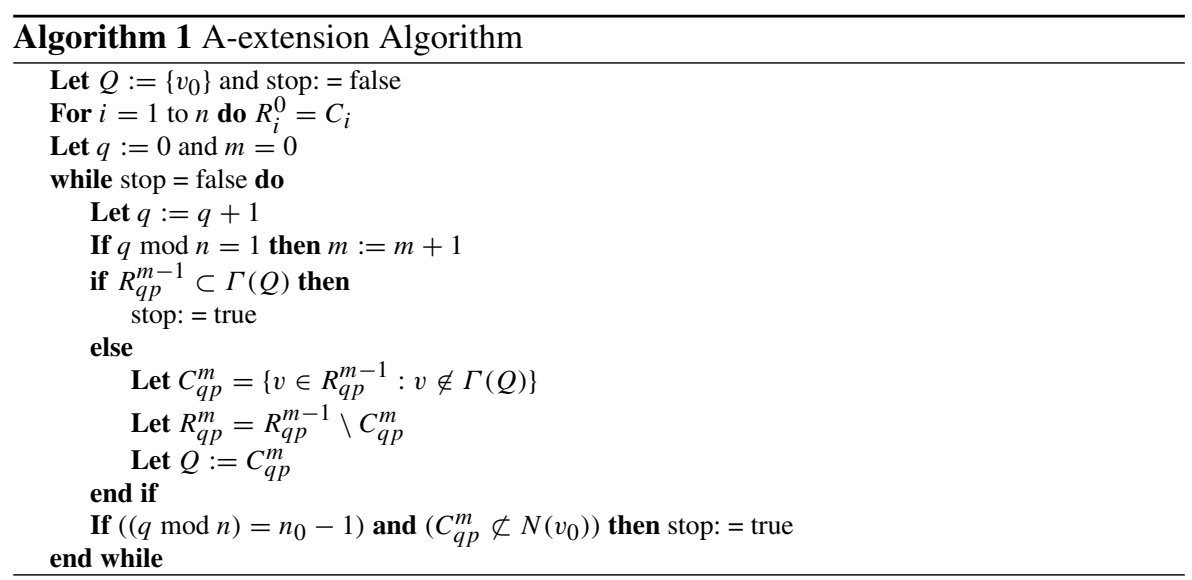

Proof We show that the A-extension algorithm allows to define a tight clique-circulant satisfying the conditions of Lemma 17.

By construction, as long as stop $=$ false, for each $m$ and $i$, the following statements hold:

(i) if $i \neq p$ then $C_{i}^{m}=\left\{v \in R_{i}^{m-1}: v \notin \Gamma\left(C_{i-p}^{m}\right)\right\}$

(ii.a) if $i=p$ and $m>1$ then $C_{p}^{m}=\left\{v \in R_{p}^{m-1}: v \notin \Gamma\left(C_{n}^{m-1}\right)\right\}$

(ii.b) if $i=p$ and $m=1$ then $C_{p}^{1}=\left\{v \in R_{p}^{0}: v \notin N\left(v_{0}\right)\right\}$

(iii) $R_{i}^{m}=R_{i}^{m-1} \backslash C_{i}^{m}$

where $R_{i}^{0}=C_{i}$, for each $i$.

It follows that $C_{i}=R_{i}^{m} \cup C_{i}^{m} \cup C_{i}^{m-1} \ldots C_{i}^{1}$, with pairwise disjoint subsets. Also, the algorithm will terminate. In fact, $v_{0}$ is of type $A$ and $C_{\left(n_{0}-1\right) p} \not \subset N\left(v_{0}\right)$. Thus, if the algorithm does not terminate before (i.e. because one of $C_{i}^{m}=\emptyset$ ), then it will terminate because there exists a finite $m$ such that $C_{\left(n_{0}-1\right) p}^{m} \not \subset N\left(v_{0}\right)$. Claim 9 and Claim 11 proves that in both situations, the Lemma 17 holds.

In the following, we say that a vertex $u \in R_{i}^{m-1}$ with $i \neq p$ is marked by a vertex $v \in C_{i-p}^{m}$ if $(u, v) \notin E(G)$ (analogously if $i=p$ and either $m>1, u \in R_{p}^{m-1}$ and $v \in C_{n}^{m-1}$ or $m=1, u \in C_{p}$ and $\left.v=v_{0}\right)$.

Claim 8 Let $v \in C_{q p}^{k}$ for some $k \geq 1$. The following statements hold:

if qp $\bmod n \neq 0$ then $(w, v) \notin E(G)$ for all $w \in C_{(q+1) p}^{1} \cup \cdots \cup C_{(q+1) p}^{k-1}$.

if qp $\bmod n=0$ then $(w, v) \notin E(G)$ for all $w \in C_{(q+1) p}^{1} \cup \cdots \cup C_{(q+1) p}^{k}$.

Proof Suppose the contrary. Let $K_{l}, K_{r}$ be a partition of $N(v)$ into two cliques. From statement (ii) of Lemma 9 we know that $w \in K_{r}$. Moreover, there exists $u$ that has marked $w$. Observe that either $u \in C_{q p}^{k^{\prime}}$ for some $k^{\prime}<k$ or $u=v_{0}$. In the former case, $u$ has been marked by some $x \in C_{(q-1) p}$ or by $x=v_{0}$ such that $u x \notin E$; observe that $v \in N(x)$ (otherwise $v \in C_{q p}^{k^{\prime}}$ ). Again from statement (ii) of Lemma 9, $x \in K_{l}$. But this is a contradiction, since $u \in N(v)$ but $u \notin K_{r}$ and $u \notin K_{l}$. The same holds 
when $u=v_{0}$, since $v_{0}$ is not complete to $C_{(q-1) p+1}=C_{n-p+1}$ which is a subset of $K_{l}$. (end of the claim)

Claim 9 If the algorithm is terminated since $R_{(q+1) p}^{m-1} \subset \Gamma(Q)$ for some $m$ and $q$, then there exists a tight clique-circulant $C^{*}$ such that $v_{0} \in V\left(C^{*}\right)$ and $V(C) \cup D(C) \subseteq$ $V\left(C^{*}\right) \cup D\left(C^{*}\right)$.

Proof Observe that, by construction, either: $Q=C_{q p}^{m}$ with $q p \bmod n \neq 0$; or $Q=$ $C_{q p}^{m-1}$ if $q p \bmod n=0$ (and in this case $m>1$ ).

In any case let $Q^{*}=Q$. Also, for all $i=1, \ldots, n$, let $C_{i}^{\prime}$ be the set of vertices from $C_{i}$ that have been marked so far when stop becomes true. Let $G^{\prime}=G\left[C_{1}^{\prime} \cup \ldots \cup C_{n}^{\prime}\right]$.

Each set $C_{i}^{\prime}$ is not empty. In fact, since $v_{0} \in A(C), C_{p}^{1} \neq \varnothing$ and by induction we have that $C_{i}^{1} \neq \varnothing$ for all $i=1, \ldots, n$. Also, either by construction or because of Claim 8, for all $i=1, \ldots, n, \Gamma\left(C_{i}^{1}\right) \cap C_{i+p}^{1}=\emptyset$. Hence, the sets $C_{1}^{\prime}, \ldots, C_{n}^{\prime}$ define an $(n, p)$-partition of $V\left(G^{\prime}\right)$.

From Claim 8, we have that $\delta\left(Q^{*}, C_{(q+1) p}^{\prime}\right)=\emptyset$. Therefore, by "backtracking" the algorithm from $Q^{*}$ to $C_{(q+1)}^{\prime}$, it is possible to find an $(n, p)$-circulant. Now applying Lemma 13 , we know that there exists $\bar{C} \in C_{G^{\prime}}(n, p)$ with a corresponding $(n, p)$-partition $\left\{\bar{C}_{i} \subseteq C_{i}^{\prime}, i=1, \ldots, n\right\}$ such that $V\left(G^{\prime}\right)=V\left(C^{\prime}\right) \cup D\left(C^{\prime}\right)$.

We claim that $V(C) \cup D(C) \subseteq V(\bar{C}) \cup D(\bar{C})$. In fact, for all $v \in D(C) \cup\{u \in$ $\left.C_{i} \backslash C_{i}^{\prime}, i=1 \ldots n\right\}, v \in D(\bar{C})$. Indeed, if $v \in D(C)$, then $|\Gamma(v, \bar{C})| \geq|\Gamma(v, C)|=$ $2 p$ and so $v \in D(\bar{C})$. Whereas if $v \in C_{i} \backslash C_{i}^{\prime}$, then $|\Gamma(v, \bar{C})| \geq 2 p-1$ (since $\left\{C_{i}, i=1, \ldots, n\right\}$ is an $(n, p)$-partition), but by construction $v \in \Gamma\left(C_{(q-1) p}^{\prime}\right)$ so $|\Gamma(v, \bar{C})| \geq 2 p$ and thus $v \in D(\bar{C})$.

Finally, since the algorithm does not terminate before, it follows that $v_{0}$ is totally linked to $\bar{C}_{\left(n_{0}-1\right) p} \subseteq C_{\left(n_{0}-1\right) p}^{\prime}$ and thus $v_{0} \in B(\bar{C})$. Hence, by the Lemma 16, it is possible to define a tight clique-circulant $C^{*}$ such that $v_{0} \in V\left(C^{*}\right)$ and $V(\bar{C}) \cup D(\bar{C}) \subseteq$ $V\left(C^{*}\right) \cup D\left(C^{*}\right)$. (end of the claim)

Claim 10 If $p_{0}=1$ then $C_{\left(n_{0}-1\right) p}^{1} \subset N\left(v_{0}\right)$.

Proof Observe that $p_{0}=1$ if and only if $n \bmod p=p-1$. In this case, if $C_{\left(n_{0}-1\right) p}^{1} \not \subset$ $N\left(v_{0}\right)$, there would exist a stable set $S$ of size $\alpha(C)$ in $G\left[\left\{C_{i}: C_{i} \not \subset N(v)\right\}\right]$ and then $\alpha\left(C \cup v_{0}\right)>\alpha(C)$. (end of the claim)

Claim 11 If the algorithm terminates because $C_{q p}^{m} \not \subset N\left(v_{0}\right)$ with $q \bmod n=n_{0}-1$, then there exists a tight clique-circulant $C^{\prime}$ such that $v_{0} \in V\left(C^{\prime}\right)$ and $V(C) \cup D(C) \subseteq$ $V\left(C^{\prime}\right) \cup D\left(C^{\prime}\right)$.

Proof First of all, observe that $q \bmod n=n_{0}-1$ if and only if $q p \bmod n=n-p+1$. Let $m^{*}$ be the value of $m$ when the algorithm terminates. We define $m_{i}=m^{*}$ for $i \in\left\{q p \bmod n: q=1, \ldots, n_{0}-1\right\}$ and $m_{i}=m^{*}-1$ otherwise. We now define: 


$$
\begin{aligned}
\mathcal{Q}= & \left(\bigcup_{i=1}^{n} \bigcup_{j=m_{i}}^{1} C_{i}^{j}\right) \cup\left\{v_{0}\right\} \\
= & \left\{C_{1}^{m^{*}-1}, C_{1}^{m^{*}-2}, \ldots, C_{1}^{1}, \ldots, C_{p-1}^{m^{*}-1}, \ldots, C_{p-1}^{1}, C_{p}^{m^{*}}, C_{p}^{m^{*}-1},\right. \\
& \left.\ldots, C_{p}^{1}, C_{p+1}^{m^{*}-1}, \ldots, C_{p+1}^{1}, \ldots, C_{n}^{m^{*}-1}, \ldots, C_{n}^{1}, v_{0}\right\}
\end{aligned}
$$

Also let $V_{\mathcal{Q}}=\bigcup_{Q \in \mathcal{Q}} V(Q)$. From Claim 8, we derive that the above sets (with the given ordering) form an $\left(n^{\prime}=\left(m^{*}-1\right) n+n_{0}, p^{\prime}=\left(m^{*}-1\right) p+p_{0}\right)$-partition of $G^{\prime}=G\left[V_{\mathcal{Q}}\right]$. Moreover, there exists an $\left(n^{\prime}, p^{\prime}\right)$-circulant included in this partition since $C_{\left(n_{0}-1\right) p}^{m^{*}} \cup\left\{v_{0}\right\} \notin K(G)$. Hence using Lemma 13, we know that there exists $C^{\prime} \in C_{G}\left(n^{\prime}, p^{\prime}\right)$ such that $V_{\mathcal{Q}}=V\left(C^{\prime}\right) \cup D\left(C^{\prime}\right)$.

Now it is left to prove that $v \in D(C) \cup\left(V(C) \backslash V_{\mathcal{Q}}\right) \Longrightarrow v \in D\left(C^{\prime}\right)$.

We denote for all $i=1, \ldots, n, N_{i}=\mid\left\{C_{i+q}^{k} \in \mathcal{Q}: q=0, \ldots, p-1 ; 1 \leq k \leq\right.$ $\left.m^{*}\right\} \mid$. By construction, it is easy to see that :

$$
N_{i}=\left(m^{*}-1\right) p+\left|\left\{C_{i+q}^{m^{*}} \in \mathcal{Q}: q=0, \ldots, p-1\right\}\right|
$$

and that

$$
\left|\left\{C_{i+q}^{m^{*}} \in \mathcal{Q}: q=0, \ldots, p-1\right\}\right|= \begin{cases}p_{0} & \text { if } 1 \leq i \leq n-p+1 \\ p_{0}-1 & \text { if } n-p+1<i \leq n\end{cases}
$$

Hence it follows that :

$$
N_{i}= \begin{cases}p^{\prime} & \text { if } \quad 1 \leq i \leq n-p+1 \\ p^{\prime}-1 & \text { if } \quad n-p+1<i \leq n\end{cases}
$$

Suppose $v \in D_{i}(C)$, then $\left|\Gamma\left(v, C^{\prime}\right)\right| \geq N_{i+1}+N_{i-p+1}+\left|N(v) \cap\left\{v_{0}\right\}\right|$. Observe that if $p \leq i \leq n-p$ then $N_{i+1}+N_{i-p+1}=2 p^{\prime}$, else $N_{i+1}+N_{i-p+1}=2 p^{\prime}-1$. Hence if $p \leq i \leq n-p$, then $\left|\Gamma\left(v, C^{\prime}\right)\right| \geq 2 p^{\prime}$. Now suppose that $i \in\{n-p+$ $1, \ldots, n, 1, \ldots, p-1\}$. We know from Lemma 11 that $i \neq n$ and thus, by Lemma 10 , $v_{0} \in N(v)$. Hence $\left|\Gamma\left(v, C^{\prime}\right)\right| \geq 2 p^{\prime}$. In any case, it follows that $v \in D\left(C^{\prime}\right)$.

Let $v \in V(C) \backslash V_{\mathcal{Q}}$. First, suppose that $v \in C_{i}$ with $i \neq 1$; then by construction $v$ is complete to $\left\{v \in C_{i-p}: v\right.$ marked\}. Therefore, $\left|\Gamma\left(v, C^{\prime}\right)\right| \geq N_{i-p}+N_{i}+$ $\left|N(v) \cap\left\{v_{0}\right\}\right|$. Hence if $p+1 \leq i \leq n-p+1$, then $\left|\Gamma\left(v, C^{\prime}\right)\right| \geq 2 p^{\prime}$. If $i \in$ $\{n-p+2, \ldots, n, 1, \ldots, p-1\}$, then $v_{0} \in N(v)$ because $v_{0} \in A(C)$. If $i=p$, then $v \in N\left(v_{0}\right)$ since otherwise $v \in C_{p}^{1}$. Thus if $i \in\{n-p+2, \ldots, n, 1, \ldots, p\}$, $\left|\Gamma\left(v, C^{\prime}\right)\right| \geq 2 p^{\prime}-1+1=2 p^{\prime}$.

Suppose now that $v \in C_{1}$; then $v$ is complete to $\left\{v \in C_{n-p+1}^{h}: 1 \leq h<m^{*}\right\}$ and $v \in N\left(v_{0}\right)$. Hence $\left|\Gamma\left(v, C^{\prime}\right)\right| \geq N_{n-p+1}-1+N_{1}+1=p^{\prime}-1+p^{\prime}+1=2 p^{\prime}$. In any case, $v \in D\left(C^{\prime}\right)$. (end of the claim) 


\section{References}

1. Cheng, E., de Vries, s.: Antiweb inequalities: strength and intractability. Congressus Numerantium 152, 5-19 (2001)

2. Cheng, E., de Vries, s.: Antiweb inequalities: strength and intractability. Math. Program. 92(1), 153175 (2002)

3. Chudnovsky, M., Seymour, P.: The structure of claw-free graphs. In: Proceedings of the Bristish Combinatorial Conference, Durham (2005 to appear)

4. Chvatal, V.: On certain polytopes associated with graphs. J. Comb. Theory 18, 138-154 (1975)

5. Edmonds, J.: Maximum matching and a polyhedron with $(0,1)$ vertices. J. Res. Nat. Bur. Standards 69, 125-130 (1965)

6. Edmonds, J.: Paths, trees and flowers. Can. J. Math. 17, 449-467 (1965)

7. Eisenbrand, F., Oriolo, G., Stauffer, G., Ventura, P.: Circular one matrices and the stable set polytope of quasi-line graphs. In: Proceedings of IPCO, pp. 291-305 (2005)

8. Galluccio, A., Sassano, A.: The rank facets of the stable set polytope for claw-free graphs. J. Comb. Theory 69, 1-38 (1997)

9. Giles, R., Trotter, L.E. Jr.: On stable set polyhedra for $k_{(1,3)}$-free graphs. J. Comb. Theory 31, 313326 (1981)

10. Grötschel, M., Lovász, L., Schrijver, A.: Geometric Algorithms and Combinatorial Optimization. Springer, Heidelberg (1988)

11. Liebling, T.M., Oriolo, G., Spille, G., Stauffer, G.: On the non-rank facets of the stable set polytope of claw-free graphs and circulant graphs. Math. Methods Oper. Res. 59, 25-35 (2004)

12. Lovász, L., Plummer, M.: Matching Theory. North Holland, Amsterdam (1986)

13. Maurras, J.: Convex hull of the edges of a graph and near bipartite graphs. Discrete Math. 46(3), 257265 (1983)

14. Minty, G.J.: On maximal independent sets of vertices in claw-free graphs. J. Comb. Theory 28, 284304 (1980)

15. Nakamura, D., Tamura, A.: A revision of minty's algorithm for finding a maximum weighted stable set of a claw-free graph. J. Oper. Res. Soc. Jpn. 44(2), 194-2004 (2001)

16. Oriolo, G.: Clique family inequalities for the stable set polytope for quasi-line graphs. Discrete Appl. Math. 132(3), 185-201 (2004)

17. Padberg, M.: On the facial structure of set packing polyhedra. Math. Program. 5, 199-215 (1973)

18. Pulleyblank, W., Shepherd, F.: Formulations for the stable set polytope of a claw-free graph. In: Rinaldi, L.W.G. (ed.) Proceedings Third IPCO conference, pp. 267-279 (1993)

19. Rebea, A.B.: Étude des stables dans les graphes quasi-adjoints. PhD thesis, Université de Grenoble (1981)

20. Sbihi, N.: Algorithme de recherche d'un stable de cardinalité maximum dans un graphe sans étoile. Discrete Math. 29, 53-76 (1980)

21. Schrijver, A.: Combinatorial Optimization. Polyhedra and efficiency (3 volumes). Springer, Heidelberg (2003)

22. Shepherd, F.: Applying lehman's theorems to packing problems. Math. Program. 71, 353-367 (1995)

23. Trotter, L.: A class of facet producing graphs for vertex packing polyhedra. Discrete Math. 12, 373388 (1975) 